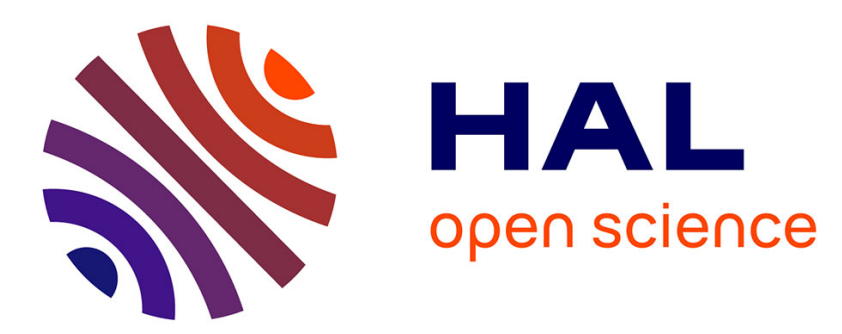

\title{
On Conditions of Oscillations and Multi-Homogeneity
}

Denis Efimov, Wilfrid Perruquetti

\section{To cite this version:}

Denis Efimov, Wilfrid Perruquetti. On Conditions of Oscillations and Multi-Homogeneity. Mathematics of Control, Signals, and Systems, 2016, 28 (3), 10.1007/s00498-015-0157-y · hal-01223154

\section{HAL Id: hal-01223154 \\ https://hal.inria.fr/hal-01223154}

Submitted on 2 Nov 2015

HAL is a multi-disciplinary open access archive for the deposit and dissemination of scientific research documents, whether they are published or not. The documents may come from teaching and research institutions in France or abroad, or from public or private research centers.
L'archive ouverte pluridisciplinaire HAL, est destinée au dépôt et à la diffusion de documents scientifiques de niveau recherche, publiés ou non, émanant des établissements d'enseignement et de recherche français ou étrangers, des laboratoires publics ou privés. 


\title{
On Conditions of Oscillations and
}

\section{Multi-Homogeneity}

\author{
Denis Efimov, Wilfrid Perruquetti
}

\begin{abstract}
The notion of homogeneity in the bi-limit from [1] is extended to local homogeneity and then to homogeneity in the multilimit. The converse Lyapunov/Chetaev theorems on (homogeneous) system instability are obtained. The problem of oscillation detection for nonlinear systems is addressed. The sufficient conditions of oscillation existence for systems homogeneous in the multi-limit are formulated. The proposed approach estimates the number of oscillating modes and the regions of their location. Efficiency of the technique is demonstrated on several examples.
\end{abstract}

Index Terms

Nonlinear systems, Homogeneity, Instability, Oscillations.

\section{INTRODUCTION}

The homogeneity is a property of nonlinear dynamical systems introduced more than fifty years ago [2], [3] meaning that the state vector rescaling does not change the system behavior. Thus, the behavior of the system trajectories on a suitably defined sphere around the origin can be extended to the whole state space. This property is used for stability analysis [1], [4], [5], [6], [7], systems approximation [8], stabilization [9], [10], [11], [12], [13], [14] and observation [1], [15]. In the works [2], [1] the homogeneity in the bi-limit is introduced, which is the homogeneity with different weights and approximating functions at a vicinity of the origin and far outside.

The global behavior of homogeneous systems is an important advantage (i.e. as in the linear case, if a homogeneous system is locally stable or unstable at the origin, then it admits this property globally). Therefore, for its (global) stability verification one can use the first (local) system approximation at the origin. If the linearization approach cannot be applied (for example, for the stable system $\dot{x}=-x^{3}$ the first order approximation gives $\dot{x}=0$, which is not suitable to make a conclusion about stability), then the Lyapunov function method can be used. It is known that homogeneous systems have homogeneous Lyapunov functions [2], [7]. For a global analysis using homogeneity, the Lyapunov function derivative sign can be checked not in the whole state space but on the sphere with the unit radius only (defined by the homogeneous norm). Additionally, the search of a homogeneous Lyapunov function may be simpler than in the common case (homogeneity restricts the kind of arguments, their

The authors are with Non-A team @ Inria, Parc Scientifique de la Haute Borne, 40 avenue Halley, 59650 Villeneuve d'Ascq, France and CRIStAL (UMR-CNRS 9189), Ecole Centrale de Lille, Cité Scientifique, 59651 Villeneuve-d'Ascq, France (denis.efimov@inria.fr, wilfrid.perruquetti@inria.fr).

D. Efimov is with Department of Control Systems and Informatics, University ITMO, 49 av. Kronverkskiy, 197101 Saint Petersburg, Russia.

This work was partially supported by the Government of Russian Federation (Grant 074-U01) and the Ministry of Education and Science of Russian Federation (Project 14.Z50.31.0031). 
relation and the function shape, while there is no method to choose Lyapunov functions in the generic case). For example, the linear system $\dot{\mathbf{x}}=\mathbf{A x}, \mathbf{x} \in \mathbb{R}^{\mathbf{n}}$ is homogeneous with the Euclidean norm, its Lyapunov function $V=\mathbf{x}^{\mathbf{T}} \mathbf{P} \mathbf{x}, \mathbf{P}^{T}=\mathbf{P}$ is a variant of the Euclidean norm. The expansion of these results on the case of instability is given in this work (converse Lyapunov/Chetaev theorems and their extensions to homogeneous systems). Recall that the Lyapunov instability theorem covers the case when the linearization has all eigenvalues with positive real part, while the Chetaev theorem (and the corresponding function) considers more general case when the linearization has some eigenvalues with positive real part [16], [17].

The global behavior of homogeneous systems simplifies their analysis, however this property restricts applicability of the approach since the most nonlinear systems (by definition) have different types of behavior depending on the state space region. To overcome this issue, the paper introduces into consideration the local homogeneity based on the bi-limit homogeneity introduced in [1], which is the existence of a homogeneous approximating (dynamical) system that coincides with the original nonlinear system on a compact set or a sphere. In this work it is shown that locally around the sphere the stability/instability property of the approximating dynamics is inherited by the original system and vice versa (for a sphere with a finite and nonzero radius). A universal formula for calculation of such a homogeneous approximating dynamics is proposed. Since the approximating dynamics is homogeneous its stability/instability can be checked using the first order approximation at the origin or using a homogeneous Lyapunov/Chetaev function (additionally, the Lyapunov/Chetaev function of the approximating dynamics has to be a local Lyapunov/Chetaev function for the original system). Therefore, to analyze the stability behavior of a nonlinear system around a sphere, linearization at the origin of an auxiliary approximating dynamical system can be used (which equations are given in the work), which is one of the main contributions.

Despite the most works are devoted to studying stability-like behavior of the origin, during recent years an interest to more complex behavior of dynamical systems, including oscillations (periodical or chaotic), has grown significantly [18], [19], [20], [21]. An important and useful concept for studying irregular oscillations is the theory introduced by V.A. Yakubovich [22], [23], [24]. Recently proposed conditions of oscillations in the sense of Yakubovich [25] are based on existence of two Lyapunov functions. The first Lyapunov function ensures local instability of the origin, while the second Lyapunov function provides global boundedness of the system trajectories, which under some mild conditions implies existence of oscillations. Such existence of two Lyapunov functions nicely interacts with homogeneity in the bi-limit: in both cases two subspaces of the system operation are considered separately. This observation serves as a motivation for proposition of conditions of oscillation existence based on the local homogeneity concept.

The main contributions of the paper are as follows. First, the notion of local homogeneity is presented based on the bi-limit homogeneity introduced in [2], [1]. Second, the conditions are proposed establishing the connection between stability/instability properties of the original nonlinear system and its local approximating dynamics. Recall that analysis (global) of nonlinear dynamical systems is a hardly solving problem, this is why local or approximate analysis is very useful and appreciated in applications. The linearization approach allows one to make a conclusion on the system behavior around a trajectory or at the point. The local homogeneity gives a similar conclusion regarding the system behavior on a sphere. It is shown that if the homogeneous approximating dynamics is stable/unstable, then the original system around the sphere has the same property. Third, the obtained stability/instability conditions are used to formulate conditions of an oscillating trajectory existence (the 
regions of oscillations in the state space are also estimated by the approach). These new conditions of oscillation existence relax the conservatism of [24], [25] and extend them to the case of several oscillating zones (when the system may be asymptotically stable around the origin and at infinity with instability regions among them). As a side of results, the necessary and sufficient instability conditions in terms of existence of Lyapunov or Chetaev functions are proposed for homogeneous systems.

The outline of the paper is as follows. The homogeneity and the oscillatory properties are introduced in Section II. Main results are presented in Section III. Some applications of the proposed results are discussed in Section IV. All proofs are given in the appendix.

\section{Preliminaries}

Let $R$ and $R_{+}$denote the sets of real and nonnegative real numbers respectively, $|x|$ and $|\mathbf{x}|$ be the absolute value of $x \in R$ and the norm of $\mathbf{x} \in R^{n}$. For a set $\mathcal{A} \subset R^{n}$ define the distance to this set from a point $\mathbf{x} \in R^{n}$ as $|\mathbf{x}|_{\mathcal{A}}=\inf \mathbf{y} \in \mathcal{A}|\mathbf{x}-\mathbf{y}|$.

Consider a nonlinear dynamical system:

$$
\dot{\mathbf{x}}=\mathbf{f}(\mathbf{x})
$$

where $\mathrm{x} \in R^{n}$ is the state vector, $\mathbf{f}: R^{n} \rightarrow R^{n}, \mathbf{f}(0)=0$ is a nonlinear continuous function such that the existence and uniqueness of solutions in forward time holds (for any initial conditions $\mathbf{x}_{0} \in R^{n}$ the solution $\mathbf{x}\left(t, \mathbf{x}_{0}\right)$ of the system (1) is defined at least locally, further we will simply write $\mathbf{x}(t)$ if origin of initial conditions is clear from the context). If for all initial conditions $\mathbf{x}_{0} \in R^{n}$ the solutions are defined for all $t \geq 0$ then the system (1) is called forward complete.

A set $\mathcal{A} \subset R^{n}$ is called forward invariant for a forward complete system (1) if for all $\mathbf{x}_{0} \in \mathcal{A}$ the property $\mathbf{x}\left(t, \mathbf{x}_{0}\right) \in \mathcal{A}$ holds for all $t \geq 0$; the set $\mathcal{A} \subset R^{n}$ is called backward invariant if for all $\mathbf{x}_{0} \in \mathcal{A}$ the property $\mathbf{x}\left(t, \mathbf{x}_{0}\right) \in \mathcal{A}$ holds for all $t \leq 0$; a set $\mathcal{A}$ is called invariant if it is simultaneously forward and backward invariant.

Recall that a continuous function $\alpha: R_{+} \rightarrow R_{+}$belongs to the class $\mathcal{K}$ if $\alpha(0)=0$ and the function is strictly increasing. The function $\alpha: R_{+} \rightarrow R_{+}$belongs to the class $\mathcal{K}_{\infty}$ if $\alpha \in \mathcal{K}$ and it is increasing to infinity. The notation $\overline{1, n}$ denotes the sequence of integers $1, \ldots, n$.

Following [26], [27], the system (1) is called (locally) asymptotically stable with respect to an invariant set $\mathcal{A} \subset R^{n}$, if there is an open set $\mathcal{X}, \mathcal{A} \subset \mathcal{X}$ such that the following two properties hold:

Stability. There is a function $\delta \in \mathcal{K}_{\infty}$ such that for any $\varepsilon \in R_{+}$and all $\mathbf{x}_{0} \in \mathcal{X}$ with $\left|\mathbf{x}_{0}\right|_{\mathcal{A}} \leq \delta(\varepsilon)$

$$
\left|\mathbf{x}\left(t, \mathbf{x}_{0}\right)\right|_{\mathcal{A}} \leq \varepsilon, \quad \forall t \geq 0
$$

Attractiveness. For any $r \in R_{+}$and $\varepsilon \in R_{+}$there is a $T_{r, \varepsilon} \geq 0$ such that for all $\mathbf{x}_{0} \in \mathcal{X}$ with $\left|\mathbf{x}_{0}\right|_{\mathcal{A}} \leq r$

$$
\left|\mathbf{x}\left(t, \mathbf{x}_{0}\right)\right|_{\mathcal{A}} \leq \varepsilon, \quad \forall t \geq T_{r, \varepsilon}
$$

The set $\mathcal{X}$ is called the domain of asymptotic stability for the set $\mathcal{A}$. If $\mathcal{X}=R^{n}$, then such a property for the system (1) is called global asymptotic stability with respect to the set $\mathcal{A}$. If $\mathcal{A}=\{0\}$, then the usual asymptotic stability with respect to a stationary point at the origin is recovered. 


\section{A. Homogeneity}

For any $r_{i}>0, i=\overline{1, n}$ and $\lambda>0$ define the dilation matrix $\Lambda_{\mathbf{r}}=\operatorname{diag}\left\{\lambda^{r_{i}}\right\}_{i=1}^{n}$ and the vector of weights $\mathbf{r}=\left[r_{1} \ldots r_{n}\right]^{T}$. For any $r_{i}>0, i=\overline{1, n}$ a homogeneous norm can be defined as follows

$$
|\mathbf{x}|_{\mathbf{r}}=\left(\sum_{i=1}^{n}\left|x_{i}\right|^{\rho / r_{i}}\right)^{1 / \rho}, \rho \geq \max _{1 \leq i \leq n} r_{i}
$$

For any $\mathbf{x} \in R^{n}$, a homogeneous norm has to be positive definite and admit an important property that $\left|\Lambda_{\mathbf{r}} \mathbf{x}\right|_{\mathbf{r}}=\lambda|\mathbf{x}|_{\mathbf{r}}$. For all $\mathbf{x} \in R^{n}$, its Euclidean norm $|\mathbf{x}|$ is related to the homogeneous norm through two functions $\underline{\sigma}_{\mathbf{r}}, \bar{\sigma}_{\mathbf{r}} \in \mathcal{K}_{\infty}$ :

$$
\underline{\sigma}_{\mathbf{r}}\left(|\mathbf{x}|_{\mathbf{r}}\right) \leq|\mathbf{x}| \leq \bar{\sigma}_{\mathbf{r}}\left(|\mathbf{x}|_{\mathbf{r}}\right)
$$

the functions $\underline{\sigma}_{\mathbf{r}}, \bar{\sigma}_{\mathbf{r}}$ define the Euclidean norm deviations with respect to the homogeneous norm. Define

$$
S_{\mathbf{r}}=\left\{\mathbf{x} \in R^{n}:|\mathbf{x}|_{\mathbf{r}}=1\right\} .
$$

Definition 1. [2] The function $g: R^{n} \rightarrow R$ is called $\mathbf{r}$-homogeneous $\left(r_{i}>0, i=\overline{1, n}\right)$ if for any $\mathbf{x} \in R^{n}$

$$
g\left(\boldsymbol{\Lambda}_{\mathbf{r}} \mathbf{x}\right)=\lambda^{d} g(\mathbf{x})
$$

for some $d \geq 0$ and all $\lambda>0, d$ is called the homogeneous degree of $g$.

The system (1) is called $\mathbf{r}$-homogeneous $\left(r_{i}>0, i=\overline{1, n}\right)$ if for any $\mathbf{x} \in R^{n}$

$$
\mathbf{f}\left(\boldsymbol{\Lambda}_{\mathbf{r}} \mathbf{x}\right)=\lambda^{d} \boldsymbol{\Lambda}_{\mathbf{r}} \mathbf{f}(\mathbf{x})
$$

for some $d \geq-\min _{1 \leq i \leq n} r_{i}$ and all $\lambda>0, d$ is called the homogeneous degree of $\mathbf{f}$.

For a continuously differentiable function $V$ the notation $D V(\mathbf{x}) \mathbf{f}(\mathbf{x})$ stands for a directional derivative with respect to the vector field $\mathbf{f}$. If the function $V$ is Lipschitz continuous, then $D^{-} V(\mathbf{x}) \mathbf{f}(\mathbf{x})$ and $D^{+} V(\mathbf{x}) \mathbf{f}(\mathbf{x})$ are stated for lower and upper directional Dini derivatives respectively:

$$
\begin{aligned}
& D^{-} V(\mathbf{x}) \mathbf{f}(\mathbf{x})=\lim _{t \rightarrow 0^{+}} \inf \frac{V(\mathbf{x}+t \mathbf{f}(\mathbf{x}))-V(\mathbf{x})}{t} \\
& D^{+} V(\mathbf{x}) \mathbf{f}(\mathbf{x})=\lim _{t \rightarrow 0^{+}} \sup \frac{V(\mathbf{x}+t \mathbf{f}(\mathbf{x}))-V(\mathbf{x})}{t} .
\end{aligned}
$$

Theorem 1. [2], [7] For the system (1) with $\mathbf{r}$-homogeneous and continuous function $\mathbf{f}: R^{n} \rightarrow R^{n}$ the following properties are equivalent:

(i) the system (1) is (locally) asymptotically stable;

(ii) there exists a continuously differentiable $\mathbf{r}$-homogeneous Lyapunov function $V: R^{n} \rightarrow R_{+}$such that for all $\mathbf{x} \in R^{n}$ :
a) $\quad \alpha_{1}(|\mathbf{x}|) \leq V(\mathbf{x}) \leq \alpha_{2}(|\mathbf{x}|)$
b) $\quad D V(\mathbf{x}) \mathbf{f}(\mathbf{x}) \leq-\alpha(|\mathbf{x}|)$,
c) $\quad V\left(\boldsymbol{\Lambda}_{\mathbf{r}} \mathbf{x}\right)=\lambda^{k} V(\mathbf{x}) \quad \forall \lambda>0$ 
for some $\alpha_{1}, \alpha_{2} \in \mathcal{K}_{\infty}, \alpha \in \mathcal{K}$ and $k \geq 0$.

The requirement on continuity of the function $\mathbf{f}$ has been relaxed in [28].

The $\mathbf{r}$-homogeneity property used in Definition 1 and Theorem 1 is introduced for some $\mathbf{r}>0$ and all $\lambda>0$. Restricting the set of admissible values for $\lambda$ we can introduce local homogeneity (in the 0-limit or in the $\infty$-limit [1], for example).

Definition 2. The function $g: R^{n} \rightarrow R$ is called $\left(\mathbf{r}, \lambda_{0}, g_{0}\right)$-homogeneous $\left(r_{i}>0, i=\overline{1, n} ; g_{0}: R^{n} \rightarrow R ; \lambda_{0}: R^{n} \rightarrow(0,+\infty)\right.$ is a continuous function or a constant $\lambda_{0} \in R_{+} \cup\{+\infty\}$ ) if for any $\mathbf{x} \in S_{\mathbf{r}}$

$$
\lim _{\lambda \rightarrow \lambda_{0}(\mathbf{x})} \lambda^{-d_{0}} g\left(\boldsymbol{\Lambda}_{\mathbf{r}} \mathbf{x}\right)-g_{0}(\mathbf{x})=0
$$

for some $d_{0} \geq 0$ (uniformly on $S_{\mathbf{r}}$ for the case $\lambda_{0} \in\{0,+\infty\}$ ).

The vector function $\mathbf{f}: R^{n} \rightarrow R^{n}$ is called $\left(\mathbf{r}, \lambda_{0}, \mathbf{f}_{0}\right)$-homogeneous $\left(r_{i}>0, i=\overline{1, n} ; \mathbf{f}_{0}: R^{n} \rightarrow R^{n} ; \lambda_{0}: R^{n} \rightarrow(0,+\infty)\right.$ is a continuous function or a constant $\left.\lambda_{0} \in R_{+} \cup\{+\infty\}\right)$ if for any $\mathbf{x} \in S_{\mathbf{r}}$

$$
\lim _{\lambda \rightarrow \lambda_{0}(\mathbf{x})} \lambda^{-d_{0}} \boldsymbol{\Lambda}_{\mathbf{r}}^{-1} \mathbf{f}\left(\boldsymbol{\Lambda}_{\mathbf{r}} \mathbf{x}\right)-\mathbf{f}_{0}(\mathbf{x})=0
$$

for some $d_{0} \geq-\min _{1 \leq i \leq n} r_{i}$ (uniformly on $S_{\mathbf{r}}$ for the case $\lambda_{0} \in\{0,+\infty\}$ ).

The system (1) is called $\left(\mathbf{r}, \lambda_{0}, \mathbf{f}_{0}\right)$-homogeneous if $\mathbf{f}$ has this property.

In the paper [1] this definition has been introduced for $\lambda_{0}=0$ and $\lambda_{0}=+\infty$ (the function $g$ is called homogeneous in the bi-limit if it is simultaneously $\left(\mathbf{r}_{0}, 0, g_{0}\right)$-homogeneous and $\left(\mathbf{r}_{\infty},+\infty, g_{\infty}\right)$-homogeneous), the case $\lambda_{0}=0$ has also been treated in [4], [5], [6], [7]. Note that the system (1) can also be homogeneous in more than two limits (some examples are considered in Section 4). Define $\boldsymbol{\Lambda}_{\mathbf{r}, 0}(\mathbf{x})=\operatorname{diag}\left\{\lambda_{0}^{r_{i}}(\mathbf{x})\right\}_{i=1}^{n}$, if $\lambda_{0}$ is a function or a constant $0<\lambda_{0}<+\infty$, then the function $g_{0}$ (respectively $\mathbf{f}_{0}$ ) is an approximation of $g$ (respectively $\mathbf{f}$ ) on the compact set

$$
\mathcal{S}=\left\{\boldsymbol{\Lambda}_{\mathbf{r}, 0}(\mathbf{x}) \mathbf{x}, \mathbf{x} \in S_{\mathbf{r}}\right\}
$$

In the following a function $g$ (respectively the system (1)) is called homogeneous in the multi-limit if there exists a finite number of triplets $\left(\mathbf{r}_{i}, \lambda_{i}, g_{i}\right.$ (respectively $\left.f_{i}\right)$ ) for which the function (respectively the system $(1)$ ) is $\left(\mathbf{r}_{i}, \lambda_{i}, g_{i}\right.$ (respectively $\left.f_{i}\right)$ ) locally homogeneous for each index $i$.

If the pairs of functions $g, g_{0}$ and $\mathbf{f}, \mathbf{f}_{0}$ are continuous, then for any $\varepsilon>0$ there exists two functions $\underline{\lambda}_{\varepsilon}(\mathbf{x}) \leq \lambda_{0}(\mathbf{x}) \leq \bar{\lambda}_{\varepsilon}(\mathbf{x})$ such that

$$
\sup _{\lambda \in\left(\underline{\lambda}_{\varepsilon}(\mathbf{x}), \bar{\lambda}_{\varepsilon}(\mathbf{x})\right), \mathbf{x} \in S_{\mathbf{r}}}\left|\lambda^{-d_{0}} g\left(\boldsymbol{\Lambda}_{\mathbf{r}} \mathbf{x}\right)-g_{0}(\mathbf{x})\right| \leq \varepsilon, \sup _{\lambda \in\left(\underline{\lambda}_{\varepsilon}(\mathbf{x}), \bar{\lambda}_{\varepsilon}(\mathbf{x})\right), \mathbf{x} \in S_{\mathbf{r}}}\left|\lambda^{-d_{0}} \boldsymbol{\Lambda}_{\mathbf{r}}^{-1} \mathbf{f}\left(\boldsymbol{\Lambda}_{\mathbf{r}} \mathbf{x}\right)-\mathbf{f}_{0}(\mathbf{x})\right| \leq \varepsilon .
$$

The coefficients $r_{i}>0, i=\overline{1, n}$ are called the weights, $d_{0}$ is the degree of homogeneity, $\mathbf{f}_{0}$ or $g_{0}$ are the approximating functions.

The following formulas give an example for the choice of locally approximating functions for any $0<\lambda_{0}(\mathbf{x})<+\infty$ and 
$\mathbf{x} \in S_{\mathbf{r}}:$

$$
g_{0}(\mathbf{x})=\lambda_{0}^{-d_{0}}(\mathbf{x}) g\left(\boldsymbol{\Lambda}_{\mathbf{r}, 0}(\mathbf{x}) \mathbf{x}\right), \mathbf{f}_{0}(\mathbf{x})=\lambda_{0}^{-d_{0}}(\mathbf{x}) \boldsymbol{\Lambda}_{\mathbf{r}, 0}^{-1}(\mathbf{x}) \mathbf{f}\left(\boldsymbol{\Lambda}_{\mathbf{r}, 0}(\mathbf{x}) \mathbf{x}\right)
$$

By construction, the limit relations from Definition 2 are satisfied for any $0<\lambda_{0}(\mathbf{x})<+\infty$ :

$$
\lim _{\lambda \rightarrow \lambda_{0}(\mathbf{x})} \lambda^{-d_{0}} g\left(\boldsymbol{\Lambda}_{\mathbf{r}} \mathbf{x}\right)-g_{0}(\mathbf{x})=0, \lim _{\lambda \rightarrow \lambda_{0}(\mathbf{x})} \lambda^{-d_{0}} \boldsymbol{\Lambda}_{\mathbf{r}, 0}^{-1} \mathbf{f}\left(\boldsymbol{\Lambda}_{\mathbf{r}} \mathbf{x}\right)-\mathbf{f}_{0}(\mathbf{x})=0
$$

Moreover, the approximating functions can be chosen homogeneous:

$$
\begin{aligned}
& g_{0}(\mathbf{x})=|\mathbf{x}|_{\mathbf{r}}^{d} \lambda_{0}^{-d_{0}}\left(\boldsymbol{\Lambda}_{|\mathbf{x}|}^{-1} \mathbf{x}\right) g\left(\boldsymbol{\Lambda}_{\mathbf{r}, 0}\left(\boldsymbol{\Lambda}_{|\mathbf{x}|}^{-1} \mathbf{x}\right) \boldsymbol{\Lambda}_{|\mathbf{x}|}^{-1} \mathbf{x}\right) \\
& \mathbf{f}_{0}(\mathbf{x})=|\mathbf{x}|_{\mathbf{r}}^{d} \lambda_{0}^{-d_{0}}\left(\boldsymbol{\Lambda}_{|\mathbf{x}|}^{-1} \mathbf{x}\right) \boldsymbol{\Lambda}_{|\mathbf{x}|} \boldsymbol{\Lambda}_{\mathbf{r}, 0}^{-1}\left(\boldsymbol{\Lambda}_{|\mathbf{x}|}^{-1} \mathbf{x}\right) \mathbf{f}\left(\boldsymbol{\Lambda}_{\mathbf{r}, 0}\left(\boldsymbol{\Lambda}_{|\mathbf{x}|}^{-1} \mathbf{x}\right) \boldsymbol{\Lambda}_{|\mathbf{x}|}^{-1} \mathbf{x}\right)
\end{aligned}
$$

where $\boldsymbol{\Lambda}_{|\mathbf{x}|}=\operatorname{diag}\left\{|\mathbf{x}|_{\mathbf{r}}^{r_{i}}\right\}_{i=1}^{n}$, provided that the degree $d$ is chosen high enough such that $g_{0}(0)$ and $\mathbf{f}_{0}(0)$ are well defined. Note that $\boldsymbol{\Lambda}_{\left|\boldsymbol{\Lambda}_{\mathbf{r}} \mathbf{x}\right|}^{-1}=\boldsymbol{\Lambda}_{\mathbf{r}}^{-1} \boldsymbol{\Lambda}_{|\mathbf{x}|}^{-1}$, then straightforward calculations show that

$$
\begin{gathered}
g_{0}\left(\boldsymbol{\Lambda}_{\mathbf{r}} \mathbf{x}\right)=\lambda^{d}|\mathbf{x}|_{\mathbf{r}}^{d} \lambda_{0}^{-d_{0}}\left(\boldsymbol{\Lambda}_{\mathbf{r}}^{-1} \boldsymbol{\Lambda}_{|\mathbf{x}|}^{-1} \boldsymbol{\Lambda}_{\mathbf{r}} \mathbf{x}\right) g\left(\boldsymbol{\Lambda}_{\mathbf{r}, 0}\left(\boldsymbol{\Lambda}_{\mathbf{r}}^{-1} \boldsymbol{\Lambda}_{|\mathbf{x}|}^{-1} \boldsymbol{\Lambda}_{\mathbf{r}} \mathbf{x}\right) \boldsymbol{\Lambda}_{\mathbf{r}}^{-1} \boldsymbol{\Lambda}_{|\mathbf{x}|}^{-1} \boldsymbol{\Lambda}_{\mathbf{r}} \mathbf{x}\right)=\lambda^{d} g_{0}(\mathbf{x}) \\
\mathbf{f}_{0}\left(\boldsymbol{\Lambda}_{\mathbf{r}} \mathbf{x}\right)=\lambda^{d}|\mathbf{x}|_{\mathbf{r}}^{d} \lambda_{0}^{-d_{0}}\left(\boldsymbol{\Lambda}_{\mathbf{r}}^{-1} \boldsymbol{\Lambda}_{|\mathbf{x}|}^{-1} \boldsymbol{\Lambda}_{\mathbf{r}} \mathbf{x}\right) \boldsymbol{\Lambda}_{\mathbf{r}} \boldsymbol{\Lambda}_{|\mathbf{x}|} \boldsymbol{\Lambda}_{\mathbf{r}, 0}^{-1}\left(\boldsymbol{\Lambda}_{\mathbf{r}}^{-1} \boldsymbol{\Lambda}_{|\mathbf{x}|}^{-1} \boldsymbol{\Lambda}_{\mathbf{r}} \mathbf{x}\right) \mathbf{f}\left(\boldsymbol{\Lambda}_{\mathbf{r}, 0}\left(\boldsymbol{\Lambda}_{\mathbf{r}}^{-1} \boldsymbol{\Lambda}_{|\mathbf{x}|}^{-1} \boldsymbol{\Lambda}_{\mathbf{r}} \mathbf{x}\right) \boldsymbol{\Lambda}_{\mathbf{r}}^{-1} \boldsymbol{\Lambda}_{|\mathbf{x}|}^{-1} \boldsymbol{\Lambda}_{\mathbf{r}} \mathbf{x}\right)=\lambda^{d} \boldsymbol{\Lambda}_{\mathbf{r}} \mathbf{f}_{0}(\mathbf{x})
\end{gathered}
$$

Note that for the chosen degrees $d$ and $d_{0}$, a continuous function $\mathbf{f}$ (respectively $g$ ) has a unique homogeneous approximation $\mathbf{f}_{0}$ (respectively $g_{0}$ ) at $0<\lambda_{0}<+\infty$. Indeed, assume that there are two homogeneous approximations $\mathbf{f}_{1}$ and $\mathbf{f}_{2}$ of $\mathbf{f}$ with the same $\mathbf{r}, d, d_{0}$ and $0<\lambda_{0}(\mathbf{x})<+\infty$, then from the definition $\mathbf{f}_{1}(\mathbf{x})=\mathbf{f}_{2}(\mathbf{x})$ for all $\mathbf{x} \in S_{\mathbf{r}}$ : since they are homogeneous we have $\mathbf{f}_{1}\left(\boldsymbol{\Lambda}_{\mathbf{r}} \mathbf{x}\right)=\lambda^{d} \boldsymbol{\Lambda}_{\mathbf{r}} \mathbf{f}_{1}(\mathbf{x})=\lambda^{d} \boldsymbol{\Lambda}_{\mathbf{r}} \mathbf{f}_{2}(\mathbf{x})=\mathbf{f}_{2}\left(\boldsymbol{\Lambda}_{\mathbf{r}} \mathbf{x}\right)$ for all $\mathbf{x} \in S_{\mathbf{r}}$ and any $\lambda>0$.

The proposed formulas (2) do not cover two limit cases with $\lambda_{0}=0$ and $\lambda_{0}=+\infty$. For the case $\lambda_{0}=0$ at least one variant of the approximating dynamics can be pointed out for differentiable functions $g$ and $\mathbf{f}$ with the property $g(0)=0$, $\mathbf{f}(0)=0$ : $g_{0}(\mathbf{x})=g^{\prime}(0) \mathbf{x}, \mathbf{f}_{0}(\mathbf{x})=\mathbf{f}^{\prime}(0) \mathbf{x}$ for $r_{i}=1, i=\overline{1, n}$ (due to linearity the approximating functions are homogeneous). Indeed, in this case

$$
g\left(\boldsymbol{\Lambda}_{\mathbf{r}} \mathbf{x}\right)=\lambda\left\{\lambda^{-1}[g(0+\lambda \mathbf{x})-g(0)]\right\}, \mathbf{f}\left(\boldsymbol{\Lambda}_{\mathbf{r}} \mathbf{x}\right)=\lambda\left\{\lambda^{-1}[\mathbf{f}(0+\lambda \mathbf{x})-\mathbf{f}(0)]\right\}
$$

and the required limit relation (in Definition 2) holds for $\lambda \rightarrow 0$ and $d_{0}=1$ since

$$
\lim _{\lambda \rightarrow 0} \lambda^{-1}[g(0+\lambda \mathbf{x})-g(0)]=g^{\prime}(0) \mathbf{x}, \lim _{\lambda \rightarrow 0} \lambda^{-1}[\mathbf{f}(0+\lambda \mathbf{x})-\mathbf{f}(0)]=\mathbf{f}^{\prime}(0) \mathbf{x}
$$

Thus in the equilibria the local homogeneity approach always may provide results similar to the linearization technique. However, opposite to linearization (that is unique) the system may have several local homogeneous approximations, some of them may provide better information about the system properties. To illustrate this claim consider the system $\dot{x}=-x^{3}+x^{4}-x^{5}$. This system has an equilibrium at the origin, its linearization has the form $\dot{x}=0$, which does not give an inside look on the system behavior around the point $x=0$. However, for $r=1$ the system also has local homogeneous approximations $\dot{x}=-x^{3}$ for $d_{0}=3, \lambda_{0}=0$ and $\dot{x}=-x^{5}$ for $d_{1}=5, \lambda_{1}=+\infty$. These systems are asymptotically stable. Recalling the results of two theorems presented below, we can conclude that the original system is stable at the origin and far outside. 
Theorem 2. [2], [8], [7] Let the system (1) be (r,0, $\left.\mathbf{f}_{0}\right)$-homogeneous with the continuous functions $\mathbf{f}: R^{n} \rightarrow R^{n}$ and $\mathbf{f}_{0}: R^{n} \rightarrow R^{n}$. If the system $\dot{\mathbf{x}}=\mathbf{f}_{0}(\mathbf{x})$ is (locally) asymptotically stable, then the system (1) is also locally asymptotically stable.

Theorem 3. [2], [1] Let the system (1) be $\left(\mathbf{r},+\infty, \mathbf{f}_{\infty}\right)$-homogeneous with the continuous functions $\mathbf{f}: R^{n} \rightarrow R^{n}$ and $\mathbf{f}_{\infty}: R^{n} \rightarrow R^{n}$. If the system $\dot{\mathbf{x}}=\mathbf{f}_{\infty}(\mathbf{x})$ is globally asymptotically stable, then there exists a compact invariant set $X_{\infty} \subset R^{n}$ containing the origin such that the system (1) is globally asymptotically stable with respect to the set $X_{\infty}$.

The theorems 2 and 3 present results on the system (1) stability derived from the corresponding properties of the approximating systems for $\lambda_{0}=0$ or $\lambda_{0}=+\infty$. The converse Lyapunov theorem similar to Theorem 1 for the homogeneous in the bi-limit systems can also be found in [1].

\section{B. Conditions of oscillations}

The function $g: R^{n} \rightarrow R$ is called monotone if the condition $x_{1} \leq x_{1}^{\prime}, \ldots, x_{n} \leq x_{n}^{\prime}$ implies that everywhere either $g\left(x_{1}, \ldots, x_{n}\right) \leq g\left(x_{1}^{\prime}, \ldots, x_{n}^{\prime}\right)$ or $g\left(x_{1}, \ldots, x_{n}\right) \geq g\left(x_{1}^{\prime}, \ldots, x_{n}^{\prime}\right)$.

Definition 3. [22], [25] For $-\infty<\pi^{-}<\pi^{+}<+\infty$ the solution $\mathbf{x}\left(t, \mathbf{x}_{0}\right)$ with $\mathbf{x}_{0} \in R^{n}$ of the system (1) is called $\left[\pi^{-}, \pi^{+}\right]-$ oscillation with respect to the output $\psi=\eta(\mathbf{x})$ (where $\eta: R^{n} \rightarrow R$ is a continuous monotone function) if the solution is defined for all $t \geq 0$ and

$$
\lim \inf _{t \rightarrow+\infty} \psi(t)=\pi^{-}, \lim \sup _{t \rightarrow+\infty} \psi(t)=\pi^{+}
$$

The solution $\mathbf{x}\left(t, \mathbf{x}_{0}\right)$ with $\mathbf{x}_{0} \in R^{n}$ of the system (1) is called oscillating, if there exist some output $\psi$ and constants $\pi^{-}, \pi^{+}$such that $\mathbf{x}\left(t, \mathbf{x}_{0}\right)$ is $\left[\pi^{-}, \pi^{+}\right]$-oscillation with respect to the output $\psi$. The forward complete system (1) is called oscillatory, if for almost all $\mathbf{x}_{0} \in R^{n}$ the solutions $\mathbf{x}\left(t, \mathbf{x}_{0}\right)$ of the system are oscillating. The oscillatory system (1) is called uniformly oscillatory, if for almost all $\mathbf{x}_{0} \in R^{n}$ for corresponding solutions $\mathbf{x}\left(t, \mathbf{x}_{0}\right)$ there exist output $\psi$ and constants $\pi^{-}$, $\pi^{+}$independently on initial conditions.

In other words the solution $\mathbf{x}\left(t, \mathbf{x}_{0}\right)$ is oscillating if the output $\psi(t)=\eta\left(\mathbf{x}\left(t, \mathbf{x}_{0}\right)\right)$ is asymptotically bounded and there is no single limit value of $\psi(t)$ for $t \rightarrow+\infty$. Note that the term "almost all solutions" is used to emphasize that generally the system (1) has a nonempty set of equilibrium points, thus there exists a set of initial conditions with zero measure such that the corresponding solutions are not oscillating. The notion of oscillations in the sense of Yakubovich is rather generic, it includes periodical oscillations (limit cycles), quasi-periodical, recurrent and chaotic trajectories. An oscillating trajectory in the sense of Yakubovich could be repelling. The trajectories could also be unbounded, it is required to find a function of the state vector, which is bounded and admits certain requirements introduced in Definition 3. Despite its complexity this notion has a Lyapunov characterization for a general nonlinear system.

Theorem 4. [25] Let the system (1) have two locally Lipschitz continuous Lyapunov functions $V_{1}$ and $V_{2}$ fulfilling the following inequalities for all $\mathrm{x} \in R^{n}$ :

$$
v_{1}(|\mathbf{x}|) \leq V_{1}(\mathbf{x}) \leq v_{2}(|\mathbf{x}|), v_{3}(|\mathbf{x}|) \leq V_{2}(\mathbf{x}) \leq v_{4}(|\mathbf{x}|), v_{1}, v_{2}, v_{3}, v_{4} \in \mathcal{K}_{\infty}
$$


and for some $0<X_{1}<v_{1}^{-1} \circ v_{2} \circ v_{3}^{-1} \circ v_{4}\left(X_{2}\right)<+\infty$ :

$$
\begin{aligned}
& D^{-} V_{1}(\mathbf{x}) \mathbf{f}(\mathbf{x})>0 \text { for all } 0<|\mathbf{x}|<X_{1} \text { and } \mathbf{x} \notin \Xi ; \\
& D^{+} V_{2}(\mathbf{x}) \mathbf{f}(\mathbf{x})<0 \text { for all }|\mathbf{x}|>X_{2} \text { and } \mathbf{x} \notin \Xi,
\end{aligned}
$$

where $\Xi \subset R^{n}$ is a set with zero Lebesgue measure containing all equilibria of the system, and

$$
\Omega \cap \Xi=\emptyset, \Omega=\left\{\mathbf{x}: v_{2}^{-1} \circ v_{1}\left(X_{1}\right) \leq|\mathbf{x}| \leq v_{3}^{-1} \circ v_{4}\left(X_{2}\right)\right\}
$$

Then the system (1) is oscillatory.

The Lyapunov function for the linearized system (1) at the origin is a candidate for the function $V_{1}$ [23]. Instead of existence of the function $V_{2}$ one can require just boundedness of the system (1) solutions with known upper bound (if this fact can be verified using another approach not dealing with a Lyapunov function analysis).

Theorem 5. [25] Let the system (1) be uniformly oscillatory with respect to the output $\psi=\eta(\mathbf{x})\left(\right.$ where $\eta: R^{n} \rightarrow R$ is a continuous function) with some $-\infty<\pi^{-}<\pi^{+}<+\infty$, and for all $\mathrm{x} \in R^{n}$ the relations

$$
\chi_{1}(|\mathbf{x}|) \leq \eta(\mathbf{x}) \leq \chi_{2}(|\mathbf{x}|), \chi_{1}, \chi_{2} \in \mathcal{K}_{\infty}
$$

are satisfied. Let the system have the only equilibrium at the origin $\left(\Xi=\left\{\mathbf{x} \in R^{n}: \mathbf{x}=0\right\}\right)$. Then there exist two locally Lipschitz continuous Lyapunov functions $V_{1}: R^{n} \rightarrow R_{+}$and $V_{2}: R^{n} \rightarrow R_{+}$such that for all $\mathbf{x} \in R^{n}$ the inequalities hold:

$$
\begin{gathered}
v_{1}(|\mathbf{x}|) \leq V_{1}(\mathbf{x}) \leq v_{2}(|\mathbf{x}|), v_{3}(|\mathbf{x}|) \leq V_{2}(\mathbf{x}) \leq v_{4}(|\mathbf{x}|), v_{1}, v_{2}, v_{3}, v_{4} \in \mathcal{K}_{\infty} ; \\
D^{-} V_{1}(\mathbf{x}) \mathbf{f}(\mathbf{x})>0 \quad \text { for all } 0<|\mathbf{x}|<\chi_{2}^{-1}\left(\pi^{-}\right) \\
D^{+} V_{2}(\mathbf{x}) \mathbf{f}(\mathbf{x})<0 \quad \text { for all }|\mathbf{x}|>\chi_{1}^{-1}\left(\pi^{+}\right) .
\end{gathered}
$$

The theorems 4 and 5 present the sufficient and necessary conditions for the system (1) to be oscillatory. Being rather simple these conditions can be useful in different applications [29], [25]. Unfortunately, in some situations these conditions could be restrictive. For example, in sufficient part they need the knowledge of two Lyapunov functions for the system (1) that can be an ambitious requirement. Additionally, the conditions are oriented to the locally unstable origin case, however, a system with several limit cycles can have a locally stable origin. These shortages can be resolved applying the homogeneity approach as it is shown below.

\section{MAIN RESULTS}

For the homogeneous in the bi-limit systems the functions $V_{1}$ and $V_{2}$ can be chosen according to the corresponding approximations at the origin or at infinity. For this purpose we are going to develop the Lyapunov theorems for locally homogeneous unstable/stable systems and apply them to detect a presence of oscillations next.

Recall that a cone in $R^{n}$ is a set consisting of half-lines emanating from some point called the vertex of the cone, in other words the set $K \subset R^{n}$ is a cone if $\lambda K \subset K$ for any $\lambda>0$, denote by $\operatorname{lb}(K)$ the lateral borders of the cone $K$. 

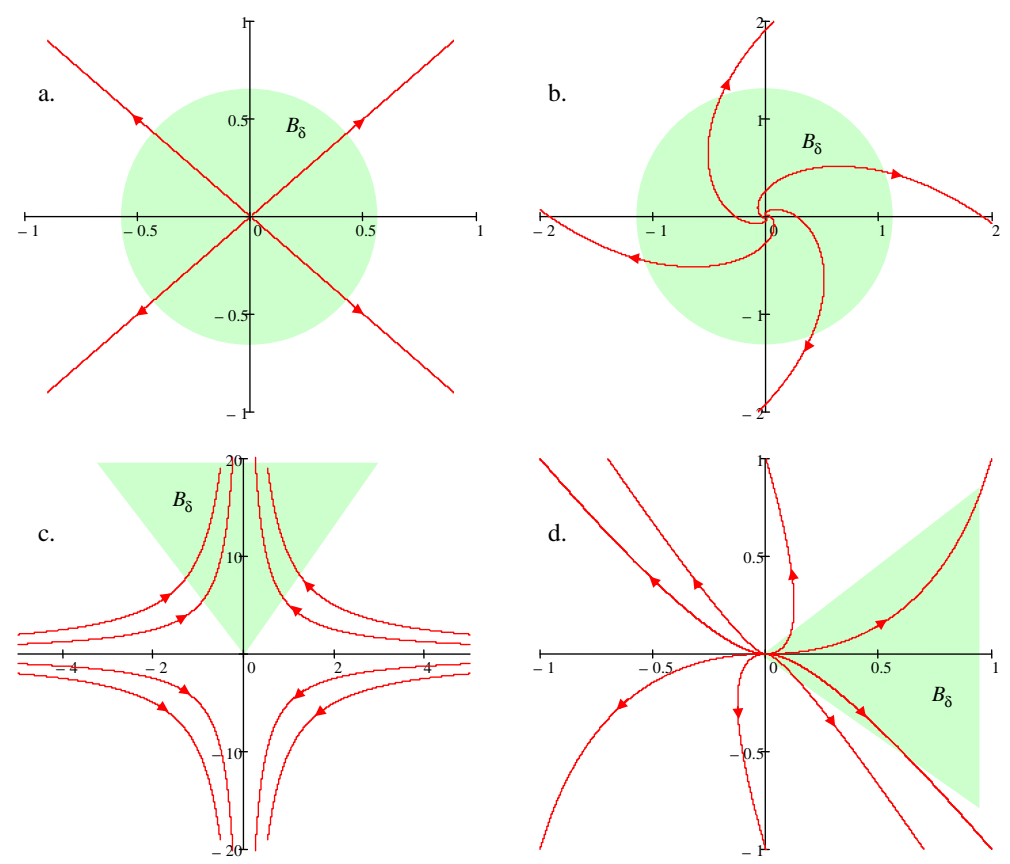

Figure 1. Illustration of basic scenarios for behavior of unstable system

\section{A. Unstable homogeneous systems}

There exist three basic setups for instability of a stationary point analysis [30], [17], a qualitative behavior in the planar case for these setups is shown in Fig. 1. First, the Lyapunov case or the case with anti-stable or strongly unstable equilibrium (when the system linearization has all roots with positive real part for example, see Fig. 1,a and Fig. 1,b), this case is studied for homogeneous systems in Lemma 1 below. Second, the case when there is a cone with all trajectories exiting from the cone basement, this situation is considered applying a Chetaev function in Lemma 2 (see Fig. 1,c). Third, the case when the cone is repulsing for all trajectories (see Fig. 1,d), again this case is covered by the Chetaev function approach (for description of Chetaev function and the approach, see Section 3.1 of [30]), the corresponding extension to homogeneous systems is presented in Lemma 3 below. The lemmas 2 and 3 may cover the cases where linearization has some eigenvalues (not necessary all) with positive real part. The necessary and sufficient Lyapunov conditions of instability can also be found in [31] (where a discontinuous Chetaev function is considered) and in [32] (where another notion of Chetaev function and idea of proof are used).

Lemma 1. For the system (1) with $\mathbf{r}-$ homogeneous and continuous function $\mathbf{f}: R^{n} \rightarrow R^{n}, \mathbf{f}(0)=0$ the following properties are equivalent:

(i) the system (1) is (locally) strongly unstable, i.e. there exists $\delta>0$ such that for any $0<\left|\mathbf{x}_{0}\right|<\delta$ there exists $T_{\mathbf{x}_{0}}>0$ such that $\left|\mathbf{x}\left(t, \mathbf{x}_{0}\right)\right|>\delta$ for all $t \geq T_{\mathbf{x}_{0}}$,

(ii) there exists a continuously differentiable $\mathbf{r}-$ homogeneous Lyapunov function $V: R^{n} \rightarrow R_{+}$such that for all $\mathbf{x} \in R^{n}$ :

a) $\quad \alpha_{1}(|\mathbf{x}|) \leq V(\mathbf{x}) \leq \alpha_{2}(|\mathbf{x}|)$

b) $\quad D V(\mathbf{x}) \mathbf{f}(\mathbf{x}) \geq \alpha(|\mathbf{x}|)$,

c) $\quad V\left(\boldsymbol{\Lambda}_{\mathbf{r}} \mathbf{x}\right)=\lambda^{k} V(\mathbf{x}) \quad \forall \lambda>0, k \geq \max _{1 \leq i \leq n} r_{i}$, 
for some $\alpha_{1}, \alpha_{2} \in \mathcal{K}_{\infty}, \alpha \in \mathcal{K}$.

Lemma 1 can be viewed as an analogue of Theorem 1 for the case of local instability of homogeneous systems (1) at the origin. It deals with the Lyapunov instability theorem. The following results present a similar extension of Chetaev theorems. For lemmas 2 and 3 we will assume that a closed cone $K \subset R^{n}$ with the vertex at $\mathbf{x}=0$ is given, denote $B_{\delta}=\{\mathbf{x} \in K: 0<|\mathbf{x}|<\delta\}$.

Lemma 2. Consider the forward complete system (1) with a Lipschitz continuous function $\mathbf{f}: R^{n} \rightarrow R^{n}, \mathbf{f}(0)=0$ and the set $B_{\delta}$ for some $\delta>0$ with all points $l b(K) \cap B_{\delta}$ being the points of entry of trajectories into $B_{\delta}$. Then the following properties are equivalent:

(i) for any $\mathbf{x}_{0} \in B_{\delta}$ there exists $T_{\mathbf{x}_{0}}>0$ such that $\left|\mathbf{x}\left(t, \mathbf{x}_{0}\right)\right|>\delta$ for $t \geq T_{\mathbf{x}_{0}}$ and $\mathbf{x}\left(t, \mathbf{x}_{0}\right) \in B_{\delta}$ for all $t \in\left[0, T_{\mathbf{x}_{0}}\right]$ (i.e. the system (1) is (locally) unstable),

(ii) there exists a continuously differentiable Chetaev function $V: R^{n} \rightarrow R_{+}$such that for all $\mathbf{x} \in B_{\delta} \cup\{0\}$ :

$$
\begin{array}{ll}
\text { a) } & \alpha_{1}(|\mathbf{x}|) \leq V(\mathbf{x}) \leq \alpha_{2}(|\mathbf{x}|) \\
\text { b) } & D V(\mathbf{x}) \mathbf{f}(\mathbf{x}) \geq \alpha_{3}(|\mathbf{x}|)
\end{array}
$$

for some $\alpha_{1}, \alpha_{2} \in \mathcal{K}_{\infty}, \alpha_{3} \in \mathcal{K}$.

For the system (1) with $\mathbf{r}-$ homogeneous function $\mathbf{f}$ the Chetaev function $V$ is $\mathbf{r}-$ homogeneous:

c) $\quad V\left(\boldsymbol{\Lambda}_{\mathbf{r}} \mathbf{x}\right)=\lambda^{k} V(\mathbf{x}) \quad \forall \lambda>0 \forall x \in R^{n}, k \geq \max _{1 \leq i \leq n} r_{i}$.

Lemma 3. Consider the forward complete system (1) with a Lipschitz continuous function $\mathbf{f}: R^{n} \rightarrow R^{n}, \mathbf{f}(0)=0$ and the set $B_{\delta}$ for some $\delta>0$ with all borders $l b(K) \cap B_{\delta}$ being the points of exit of trajectories from $B_{\delta}$ and the only invariant solution is at the origin (i.e. for all $\mathbf{x}_{0} \in B_{\delta}$ there exists $T_{\mathbf{x}_{0}} \geq 0$ such that $\left.\mathbf{x}\left(T_{\mathbf{x}_{0}}, \mathbf{x}_{0}\right) \notin B_{\delta}\right)$. Then the following properties are equivalent:

(i) the system (1) is (locally) unstable into the backward invariant set $B_{\delta}$,

(ii) there exists a continuously differentiable Chetaev function $V: R^{n} \rightarrow R_{+}$such that for all $\mathbf{x} \in B_{\delta} \cup\{0\}$ :

$$
\begin{gathered}
\text { a) } \quad \alpha_{1}(|\mathbf{x}|) \leq V(\mathbf{x}) \leq \alpha_{2}(|\mathbf{x}|) \\
\text { b) } \quad D V(\mathbf{x}) \mathbf{f}(\mathbf{x}) \geq \alpha_{3}(|\mathbf{x}|) \\
\text { for some } \alpha_{1}, \alpha_{2} \in \mathcal{K}_{\infty}, \alpha_{3} \in \mathcal{K} .
\end{gathered}
$$

For the system (1) with $\mathbf{r}$-homogeneous function $\mathbf{f}$ the Chetaev function $V$ is $\mathbf{r}-$ homogeneous:

c) $\quad V\left(\boldsymbol{\Lambda}_{\mathbf{r}} \mathbf{x}\right)=\lambda^{k} V(\mathbf{x}) \quad \forall \lambda>0 \forall x \in R^{n}, k \geq \max _{1 \leq i \leq n} r_{i}$.

The first lemma allows us for consideration of strongly unstable or anti-stable systems (for example, the systems with linearization having all eigenvalues with strictly positive real parts), lemmas 2 and 3 oriented on analysis of unstable systems where linearization may have some eigenvalues with positive real parts (saddle equilibria, for instance). In all cases existence of positive definite Chetaev functions with positive derivatives on appropriate regions $B_{\delta}$ are proven (the functions can be chosen homogeneous for $\mathbf{r}$-homogeneous systems (1)). The function $V$ established in Lemma 1 is called Lyapunov function, which is a particular case of Chetaev functions presented in lemmas 2 and 3 [16]. 


\section{B. Stability/instability conditions for locally homogeneous systems}

An advantage of the $\mathbf{r}$-homogeneous system (1) is that the global behavior of the system can be completely characterized by the behavior of the system on the sphere with the unit radius $S_{\mathbf{r}}$. To explain this property let us introduce the coordinate transformation $\mathbf{x}=\Lambda_{\mathbf{r}} \mathbf{y}$ that connects any $\mathbf{x} \in R^{n}$ with $\mathbf{y} \in S_{\mathbf{r}}$ for suitably chosen $\lambda>0$. Let $V: R^{n} \rightarrow R_{+}$be a continuously differentiable homogeneous Lyapunov function (as used in Theorem 1 and Lemma 1), then

$$
D V(\mathbf{x}) \mathbf{f}(\mathbf{x})=D V\left(\boldsymbol{\Lambda}_{\mathbf{r}} \mathbf{y}\right) \mathbf{f}\left(\boldsymbol{\Lambda}_{\mathbf{r}} \mathbf{y}\right)=\lambda^{d+k} D V(\mathbf{y}) \mathbf{f}(\mathbf{y})=|\mathbf{x}|_{\mathbf{r}}^{d+k} D V(\mathbf{y}) \mathbf{f}(\mathbf{y})
$$

where $d$ is the homogeneity degree of the function $\mathbf{f}$ and $k$ is the degree of the Lyapunov function $V$. Therefore, sign definiteness of the function $V$ derivative can be checked on the sphere $S_{\mathbf{r}}$ only.

For the $\left(\mathbf{r}, \lambda_{0}, \mathbf{f}_{0}\right)$-homogeneous system (1) this technique establishes the relation between the global stability at the origin of the approximating dynamics

$$
\dot{\mathbf{x}}=\mathbf{f}_{0}(\mathbf{x})
$$

and the local one of the original system (1). The conditions of such a relation are established below (recall that we denoted $\left.\mathcal{S}=\left\{\boldsymbol{\Lambda}_{\mathbf{r}, 0}(\mathbf{x}) \mathbf{x}, \mathbf{x} \in S_{\mathbf{r}}\right\}\right)$

Proposition 1. Let the system (1) be $\left(\mathbf{r}, \lambda_{0}, \mathbf{f}_{0}\right)$-homogeneous with $0<\lambda_{0}(\mathbf{x})<+\infty$, the functions $\mathbf{f}: R^{n} \rightarrow R^{n}$ and $\mathbf{f}_{0}: R^{n} \rightarrow R^{n}$ be continuous, the function $\mathbf{f}_{0}$ be $\mathbf{r}-$ homogeneous. Let $V_{0}: R^{n} \rightarrow R_{+}$be a $\mathbf{r}$-homogeneous, continuously differentiable and radially unbounded Lyapunov function and the set $\mathcal{S}$ be a level of $V_{0}$, i.e. $\mathcal{S}=\left\{\mathbf{x} \in R^{n}: V_{0}(\mathbf{x})=b\right\}$ for some $b>0$. Then the following properties are equivalent:

1. The approximating dynamics (3) has a Lyapunov function $V_{0}$ and $\sup _{\mathbf{y} \in S_{\mathbf{r}}} D V_{0}(\mathbf{y}) \mathbf{f}_{0}(\mathbf{y})<0\left(\inf _{\mathbf{y} \in S_{\mathbf{r}}} D V_{0}(\mathbf{y}) \mathbf{f}_{0}(\mathbf{y})>0\right)$.

2. The set $\mathcal{X}=\left\{\mathbf{x} \in R^{n}: V_{0}(\mathbf{x}) \leq b\right\}$ is locally asymptotically stable (anti-stable) for the system (1) and $\sup _{\mathbf{x} \in \mathcal{S}} D V_{0}(\mathbf{x}) \mathbf{f}(\mathbf{x})<0\left(\inf _{\mathbf{x} \in \mathcal{S}} D V_{0}(\mathbf{x}) \mathbf{f}(\mathbf{x})>0\right)$.

This result establishes the stability relation between the approximating dynamics (3) and the locally homogeneous system (1). Similar equivalence result can also be formulated for the Chetaev case of instability. Note that to apply this proposition in the direction $1 \Rightarrow 2$ it is not obligatory to find a Lyapunov function for the system (3). Indeed, according to (2) the degree of homogeneous approximation for $0<\lambda_{0}<+\infty$ always can be selected 0 . In such a case the system (3) can be rewritten as follows:

$$
\dot{\mathbf{x}}=\mathbf{f}_{0}(\mathbf{x})=\mathbf{A x}+\mathbf{B} \phi(\mathbf{H x})
$$

where $\mathbf{A} \in R^{n \times n}, \mathbf{B} \in R^{n \times m}, \mathbf{H} \in R^{p \times n}$ are matrices of appropriate dimensions and $\phi: R^{p} \rightarrow R^{m}$ is a globally Lipschitz continuous function with the Lipschitz constant $\gamma>0$, i.e. $\left|\phi(\mathbf{z})-\phi\left(\mathbf{z}^{\prime}\right)\right| \leq \gamma\left|\mathbf{z}-\mathbf{z}^{\prime}\right|$ for any $\mathbf{z}, \mathbf{z}^{\prime} \in R^{p}$. If the system (3) has a well defined linearization, then $\mathbf{B}=0$ and $\mathbf{A}=\partial \mathbf{f}_{0}(\mathbf{x}) /\left.\partial \mathbf{x}\right|_{\mathbf{x}=0}$. Asymptotic stability at the origin of Lipschitz nonlinear systems can be investigated using the linear system theory [33], [34], [35]. In particular, if there exists a solution $\mathbf{P} \in R^{n \times n}$, 
$\mathbf{P}^{T}=\mathbf{P}>0$ of the following Riccati equation

$$
\mathbf{A}^{T} \mathbf{P}+\mathbf{P A}+\mathbf{P B B}^{T} \mathbf{P}+\gamma^{2} \mathbf{H}^{T} \mathbf{H}<0
$$

then the system (3) is globally asymptotically stable. If the nonlinear function $\phi$ belongs to the class of sector nonlinearities, then the absolute stability theory and LMIs independent on $\gamma$ can be used [36]. Next, owing Proposition 1 result we can make a conclusion about stability/instability of the system (1) on the compact set $\mathcal{S}$. Therefore, this proposition opens the gates for application of the first order approximation (4) analysis for non-local investigation of nonlinear systems on a compact.

Skipping homogeneity requirement for the function $\mathbf{f}_{0}$ and including the values $\lambda_{0}=0, \lambda_{0}=+\infty$ we can formulate the following proposition.

Proposition 2. Let the system (1) be $\left(\mathbf{r}, \lambda_{0}, \mathbf{f}_{0}\right)$-homogeneous, the functions $\mathbf{f}: R^{n} \rightarrow R^{n}$ and $\mathbf{f}_{0}: R^{n} \rightarrow R^{n}$ be continuous and the approximating dynamics (3) have $\mathbf{r}$-homogeneous, continuously differentiable and radially unbounded Lyapunov function $V_{0}: R^{n} \rightarrow R_{+}$, the set $\mathcal{S}$ is a level set for $V_{0}$, i.e. $\mathcal{S}=\left\{\mathbf{x} \in R^{n}: V_{0}(\mathbf{x})=b\right\}$ for some $b \geq 0$.

$$
\text { Let } a_{s}=-\sup _{\mathbf{y} \in S_{\mathbf{r}}} D V_{0}(\mathbf{y}) \mathbf{f}_{0}(\mathbf{y}), a_{s}>0 \text {, then }
$$

1) if $\lambda_{0}=0$, then there exists $0<\epsilon<+\infty$ such that the system (1) is locally asymptotically stable at the origin with the domain of asymptotic stability containing the set

$$
X_{0}=\left\{\mathbf{x} \in R^{n}: V(\mathbf{x}) \leq \epsilon\right\}
$$

2) if $\lambda_{0}=+\infty$, then there exists $0<\epsilon<+\infty$ such that the system (1) is globally asymptotically stable with respect to the forward invariant set $X_{0}$;

3) if $0<\lambda_{0}(\mathbf{x})<+\infty$, then there exists $0<\epsilon<+\infty$ such that the system (1) is locally finite-time stable with respect to the forward invariant set $X_{1}=\left\{\mathbf{x} \in R^{n}: V(\mathbf{x}) \leq b-\epsilon\right\}$ with the region of attraction

$$
X=\left\{\mathbf{x} \in R^{n}: b-\epsilon \leq V(\mathbf{x}) \leq b+\epsilon\right\} .
$$

(ii) Let $a_{u}=\inf _{\mathbf{y} \in S_{\mathbf{r}}} D V_{0}(\mathbf{y}) \mathbf{f}_{0}(\mathbf{y}), a_{u}>0$, then

1) if $\lambda_{0}=0$, then there exists $0<\epsilon<+\infty$ such that the system (1) is asymptotically stable with respect to the forward invariant set $R^{n} \backslash X_{0}$ with the region of attraction $X_{0} \backslash\{0\}$;

2) if $\lambda_{0}=+\infty$, then there exists $0<\epsilon<+\infty$ such that the set $R^{n} \backslash X_{0}$ is forward invariant for the system (1);

3) if $0<\lambda_{0}(\mathbf{x})<+\infty$, then there exist $0<\epsilon<+\infty$ such that the system (1) is locally finite time stable with respect to the forward invariant set $X_{2}=\left\{\mathbf{x} \in R^{n}: V(\mathbf{x}) \geq b+\epsilon\right\}$ with the region of attraction $X$.

In other words the result of Proposition 2 means that the behavior of the system (1) is inherited from the behavior of (3) within the set $X$. The first two parts of the case (i) correspond to theorems 2 and 3. Proposition 2 derives more accurately than Proposition 1 the stable and unstable sets of the system (1). The conditions of the last proposition can be relaxed skipping assumptions on the homogeneity of $\mathbf{f}_{0}$ and the continuity of $\mathbf{f}, \mathbf{f}_{0}$ as follows. 
Corollary 1. Let $\mathbf{f}_{0}: R^{n} \rightarrow R^{n}$ be given and the approximating dynamics (3) have $\mathbf{r}$-homogeneous, continuously differentiable and radially unbounded Lyapunov function $V_{0}: R^{n} \rightarrow R_{+}, \mathcal{S}=\left\{\mathrm{x} \in R^{n}: V_{0}(\mathrm{x})=b\right\}$ for some $b \geq 0$. Let one of the following properties hold

(ii) $\quad a=\inf _{\mathbf{y} \in S_{\mathbf{r}}} D V_{0}(\mathbf{y}) \mathbf{f}_{0}(\mathbf{y}), a>0$,

and there exist $\underline{\lambda}_{\varepsilon}(\mathbf{y}) \leq \lambda_{0}(\mathbf{y}) \leq \bar{\lambda}_{\varepsilon}(\mathbf{y})$ such that $\sup _{\lambda \in\left(\underline{\lambda}_{\varepsilon}(\mathbf{y}), \bar{\lambda}_{\varepsilon}(\mathbf{y})\right), \mathbf{y} \in S_{\mathbf{r}}}\left|D V_{0}(\mathbf{y})\left[\lambda^{-d} \boldsymbol{\Lambda}_{\mathbf{r}}^{-1} \mathbf{f}\left(\boldsymbol{\Lambda}_{\mathbf{r}} \mathbf{y}\right)-\mathbf{f}_{0}(\mathbf{y})\right]\right| \leq \varepsilon<a$, then all claims (i),1-(i),3 or (ii),1-(ii),3 of Proposition 2 are valid.

Another way to relax the conditions of Proposition 2 consists in application of the results of lemmas 2 and 3 for instability detection at the origin (for $\lambda_{0}=0$ ).

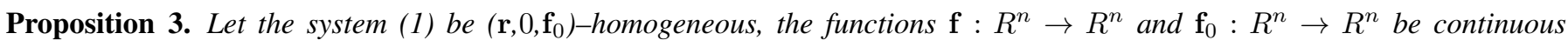
and the approximating dynamics (3) have $\mathbf{r}$-homogeneous and continuously differentiable Chetaev function $V_{0}: R^{n} \rightarrow R_{+}$, $\alpha_{1}(|\mathbf{x}|) \leq V_{0}(\mathbf{x}) \leq \alpha_{2}(|\mathbf{x}|), \alpha_{1}, \alpha_{2} \in \mathcal{K}_{\infty}$ for all $\mathbf{x} \in B_{\delta}, B_{\delta}=\left\{\mathbf{x} \in R^{n}: 0 \leq|\mathbf{x}|_{\mathbf{r}}<\delta, \mathbf{x} \in K\right\}, \delta>1$ and $K$ be a closed cone with the vertex at the origin, all points $l b(K) \cap B_{\delta} \backslash\{0\}$ be either the points of entry or the points of exit for $\mathbf{f}$ and $\mathbf{f}_{0}$. Let $a=\inf _{\mathbf{y} \in S_{\mathbf{r}} \cap K} D V_{0}(\mathbf{y}) \mathbf{f}_{0}(\mathbf{y}), a>0$, then the system (1) is unstable at the origin.

Let us apply these results for detection of oscillations.

C. Oscillations in locally homogeneous systems

Let $0 \leq \lambda_{1} \leq \ldots \leq \lambda_{N} \leq+\infty$ be an ordered sequence for a given integer $N>0$ (for brevity of formulation, the presentation in this section is given for constant $\lambda_{j}, j=\overline{1, N}$, the case with the functions $\lambda_{j}(\mathbf{x})$ can be introduced similarly).

Theorem 6. Let the system (1) be $\left(\mathbf{r}_{j}, \lambda_{j}, \mathbf{f}_{j}\right)$-homogeneous for $j=\overline{1, N}$, the functions $\mathbf{f}: R^{n} \rightarrow R^{n}$ and $\mathbf{f}_{j}: R^{n} \rightarrow R^{n}$, $j=\overline{1, N}$ be continuous and the locally approximating dynamical systems $\dot{\mathbf{x}}=\mathbf{f}_{j}(\mathbf{x}), j=\overline{1, N}$ have $\mathbf{r}_{j}$-homogeneous and continuously differentiable Lyapunov functions $V_{j}: R^{n} \rightarrow R_{+}, \alpha_{1, j}\left(|\mathbf{x}|_{\mathbf{r}_{j}}\right) \leq V_{j}(\mathbf{x}) \leq \alpha_{2, j}\left(|\mathbf{x}|_{\mathbf{r}_{j}}\right), \alpha_{1, j}, \alpha_{2, j} \in \mathcal{K}_{\infty}$ for all $\mathbf{x} \in R^{n}$ and $j=\overline{1, N}$. Let $\Xi \subset R^{n}$ be the set containing all equilibria of the system (1). Let one of the following conditions hold.

(i) $\quad$ There exists $1 \leq j^{*}<N$ such that

$$
a_{j^{*}}=\inf _{\mathbf{y} \in S_{\mathbf{r}_{j^{*}}}} D V_{j^{*}}(\mathbf{y}) \mathbf{f}_{j^{*}}(\mathbf{y})>0, a_{j^{*}+1}=-\sup _{\mathbf{y} \in S_{\mathbf{r}_{j^{*}+1}}} D V_{j^{*}+1}(\mathbf{y}) \mathbf{f}_{j^{*}+1}(\mathbf{y})>0
$$

and two sets $X_{k}=\left\{\mathbf{x} \in R^{n}: V_{k}(\mathbf{x})=b_{k}\right\} \cap\left\{\mathbf{x} \in R^{n}: \underline{\lambda}_{k} \leq|\mathbf{x}|_{\mathbf{r}_{k}} \leq \bar{\lambda}_{k}\right\}$ for $k=j^{*}, j^{*}+1$ are connected and nonempty for some $b_{k} \in\left[\alpha_{1, k}\left(\underline{\lambda}_{k}\right), \alpha_{2, k}\left(\bar{\lambda}_{k}\right)\right]$ and

$$
\sup _{\mathbf{y} \in S_{\mathbf{r}_{k}}}\left|D V_{k}(\mathbf{y})\left[\lambda^{-d} \boldsymbol{\Lambda}_{\mathbf{r}_{k}}^{-1} \mathbf{f}\left(\boldsymbol{\Lambda}_{\mathbf{r}_{k}} \mathbf{y}\right)-\mathbf{f}_{k}(\mathbf{y})\right]\right|<a_{k}
$$

for all $\lambda \in\left(\underline{\lambda}_{k}, \bar{\lambda}_{k}\right), \underline{\lambda}_{k} \leq \lambda_{k} \leq \bar{\lambda}_{k}$ (such constants $\underline{\lambda}_{k}, \bar{\lambda}_{k}$ exist due to the homogeneity assumption), and

$$
\Omega \cap \Xi=\emptyset, \Omega=\Omega_{j *} \cap \Omega_{j *+1}, \quad \Omega_{j *}=\left\{\mathbf{x} \in R^{n}: V_{j *}(\mathbf{x}) \geq b_{j *}\right\}, \Omega_{j *+1}=\left\{\mathbf{x} \in R^{n}: V_{j *+1}(\mathbf{x}) \leq b_{j *+1}\right\} .
$$




$$
a_{j^{*}}=-\sup _{\mathbf{y} \in S_{\mathbf{r}_{j^{*}}}} D V_{j^{*}}(\mathbf{y}) \mathbf{f}_{j^{*}}(\mathbf{y})>0, a_{j^{*}+1}=\inf _{\mathbf{y} \in S_{\mathbf{r}_{j^{*}+1}}} D V_{j^{*}+1}(\mathbf{y}) \mathbf{f}_{j^{*}+1}(\mathbf{y})>0
$$

and the sets $X_{k}, k=j^{*}, j^{*}+1$ are connected and nonempty where $\underline{\lambda}_{k} \leq \lambda_{k} \leq \bar{\lambda}_{k}$ are defined as for the case (i), and $\Omega \cap \Xi=\emptyset, \Omega=\Omega_{j *} \cap \Omega_{j *+1}$.

Then the system (1) has oscillating trajectories into the set $\Omega$.

The result of the last theorem implies that if the system (1) is locally homogeneous and unstable in an inner (outer) subset, and locally homogeneous and stable in an outer (inner) subset, then between these subsets should exist an invariant set containing an oscillating trajectory provided that the equilibria are excluded from this region. The conditions of Theorem 6 can be relaxed taking in mind the results of Corollary 1 or Proposition 3 (these reformulations are omitted here for brevity of presentation).

The set $\Omega$ can be used to estimate the constants $\pi^{-}, \pi^{+}$, i.e. to estimate the amplitude of oscillation.

It is worth to note that Theorem 4 deals with one oscillating zone only, it was also assumed that the origin is strongly unstable. Theorem 6 relaxes these constraints, it allows the presence of multiple oscillating zones to be detected, the origin can be stable or unstable.

\section{Procedure for oscillation detection}

In this section we are going to comment on the proposed conditions to analyze oscillating trajectories and present the procedure for their applications.

The analysis of nonlinear system for stability, instability or oscillation existence is a rather complex problem that lacks for constructive applied approaches. The class of linear systems, on the contrary, has a complete list of methods for their analysis. The class of homogeneous systems being rather generic extends the linear systems. Considering homogeneous systems it seems possible to propose constructive (applicable in practice) approaches for nonlinear systems analysis, or at least reduce the complexity of investigation. The class of locally homogeneous systems introduced here is larger than the class of homogeneous ones (for any $\mathbf{f}$ and given $\mathbf{r}, d, d_{0}$ and $0<\lambda_{0}(\mathbf{x})<+\infty$, the corresponding homogeneous approximation $\mathbf{f}_{0}$ is given in (2)). In addition, it is larger than the class of systems having a homogeneous approximation for $\lambda_{0}=0$ and/or $\lambda_{0}=+\infty$ introduced in [1], it is due to the requirement introduced in Definition 2 for the case $\lambda_{0} \in\{0,+\infty\}$ on uniformity of the limit, which may fail to exist for a continuous $\mathbf{f}$.

Two types of results are established. The first group deals with stability/instability analysis for locally homogeneous systems on the basis of their approximating dynamics. It is shown that if the approximating system (3) has corresponding Lyapunov or Chetaev function, then there exists a domain in the state space where the system (1) inherits the same stability/instability properties. If the approximating dynamics $\mathbf{f}_{0}$ is homogeneous, then the existence of homogeneous Lyapunov or Chetaev functions has been proven (Theorem 1 and lemmas $1,2,3$ ). In addition, the stability or instability of $\mathbf{f}_{0}$ on the sphere $S_{\mathbf{r}}$ can be checked at the origin by the first order approximation (4) analysis (if the system $\mathbf{f}_{0}$ is homogeneous, then it exhibits globally the same behavior, which can be classified by stability/instability at the origin). The second set of results is devoted to exposure of oscillating in the sense of Yakubovich trajectories for nonlinear systems. It is shown that if the system has 
two locally approximating dynamics, one is stable and another one is unstable in the corresponding domains, and the region between these domains does not contain equilibria, then the system is oscillating in the sense of Yakubovich. The formal procedure for application of these conditions can be described as follows:

1) Find all equilibrium points of the system (define the set $\Xi$ ).

2) Find $0 \leq \lambda_{1}(\mathbf{x}) \leq \ldots \leq \lambda_{N}(\mathbf{x}) \leq+\infty, N>0$ defining the regions of local homogeneity.

3) For each locally approximating dynamics $\mathbf{f}_{k}, k=\overline{1, N}$ it is necessary to find Lyapunov or Chetaev functions establishing local stability/instability of the system (if the functions $\mathbf{f}_{k}$ are homogeneous, then the stability/instability may be checked at the origin in the first order approximation).

4) Verify the conditions $(i)$ or (ii) of Theorem 6 (taking in mind Corollary 1 or Proposition 3).

The most complex steps of the procedure are 2 and 3. For a given nonlinear system (1), there is no common recommendation how to find $0 \leq \lambda_{1} \leq \ldots \leq \lambda_{N} \leq+\infty$ defining the partition. The partition is strongly related with the shape of $\mathbf{f}$. The formulas for approximating functions $\mathbf{f}_{0}$ for any $0<\lambda_{0}(\mathbf{x})<+\infty$ are given after Definition 2, however the issue is to find $\lambda_{0}$ and the corresponding weights $\mathbf{r}$ such that the approximating function $\mathbf{f}_{0}$ generates stable or unstable dynamics in (3).

The procedure can also be applied in a reverse way for a complex oscillating system design and for the control synthesis that provides oscillating behavior for a nonlinear system [37]. Let us test this procedure on several academic examples.

\section{EXAMPLES}

In this section we will consider two groups of examples illustrating the results of Proposition 1 (on equivalence of stability for the original system and its local homogeneous approximation) and Theorem 6 (on detection of oscillating modes).

\section{A. Examples for Proposition 1}

Consider the Lorenz model:

$$
\left\{\begin{array}{l}
\dot{x}=\sigma(y-x) \\
\dot{y}=x(\rho-z)-y, \\
\dot{z}=\quad x y-\beta z
\end{array}\right.
$$

where $\sigma>0, \rho>0$ and $\beta>0$ are the model parameters. The system has three equilibrium points $( \pm \sqrt{\beta(\rho-1)}, \pm \sqrt{\beta(\rho-1)}, 1),(0,0,0)$ and for some values of parameters a strange attractor or oscillating trajectories. Introducing the new coordinates $x_{1}=x, x_{2}=y, x_{3}=\rho-z$ we obtain the system in the form (1) for

$$
\mathbf{f}(\mathbf{x})=\left[\begin{array}{c}
\sigma\left(x_{2}-x_{1}\right) \\
x_{1} x_{3}-x_{2} \\
-x_{1} x_{2}-\beta x_{3}+\beta \rho
\end{array}\right] .
$$




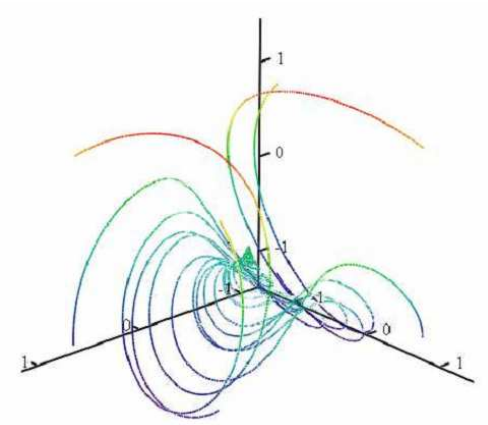

Figure 2. Trajectories of local approximation for Lorenz model

Local approximation or linearization shows that the equilibria are unstable. For $r_{1}=1, r_{2}=r_{3}=2$ and $d_{0}=1$ we obtain the following approximation of $\mathbf{f}$ for $\lambda_{0}=+\infty$ :

$$
\mathbf{f}_{0}(\mathbf{x})=\left[\begin{array}{c}
\sigma x_{2} \\
x_{1} x_{3} \\
-x_{1} x_{2}
\end{array}\right]
$$

which is a purely oscillating system (all trajectories are closed orbits), that does not allow us to make a conclusion about boundedness of trajectories of the Lorenz model. The system has a Lyapunov function $V(\mathbf{x})=0.5\left(\sigma^{-1} x_{1}^{2}+x_{2}^{2}+x_{3}^{2}\right)$ with $\dot{V} \leq 0.5 \beta \rho^{2}-0.5 \beta x_{3}^{2}-0.5 x_{2}^{2}-0.5 x_{1}^{2} \leq 0.5 \beta \rho^{2}-\mu V$, where $\mu=\min \{1, \beta\} / \max \left\{1, \sigma^{-1}\right\}$. Therefore, the function $V$ has a strictly negative derivative for $V>0.5 \beta \rho^{2} \mu^{-1}$. Let us show that the corresponding local homogeneous approximation for $V>0.5 \beta \rho^{2} \mu^{-1}$ will be stable. Choose $\mathcal{S}=\left\{\mathbf{x} \in R^{3}: V(\mathbf{x})=V_{0}\right\}$ with $V_{0}=0.5 \beta \rho^{2} \mu^{-1}+\varepsilon$ for some $\varepsilon>0$ and $r_{1}=r_{2}=r_{3}=1$, then a direct computation shows that

$$
\lambda_{0}(\mathbf{x})=\sqrt{\frac{2 \sigma V_{0}}{\left(1-x_{1}^{2}\right) \sigma+x_{1}^{2}}},
$$

which is well defined for all $\mathrm{x} \in S_{\mathbf{r}}$. From (2) we obtain the expression for the locally approximating function

$$
\mathbf{f}_{0}(\mathbf{x})=\left[\begin{array}{c}
\sigma\left(x_{2}-x_{1}\right) \\
\kappa(\mathbf{x}) x_{1} x_{3}-x_{2} \\
-\kappa(\mathbf{x}) x_{1} x_{2}-\beta x_{3}+\kappa(\mathbf{x})^{-1} \beta \rho
\end{array}\right]
$$

for $d=d_{0}=0$ and $\kappa(\mathbf{x})=\sqrt{V_{0} / V(\mathbf{x})}$. Computing the derivative of $V$ for the approximation dynamics (3) we get

$$
\dot{V} \leq 0.5 \beta \rho^{2} V / V_{0}-0.5 \beta x_{3}^{2}-0.5 x_{2}^{2}-0.5 x_{1}^{2} \leq-\varrho V
$$

where $\varrho=\mu-0.5 \beta \rho^{2} /\left(0.5 \beta \rho^{2} \mu^{-1}+\varepsilon\right)$ and $\varrho>0$ for any $\varepsilon>0$. Therefore the approximating dynamics would be globally asymptotically stable for all such $\varepsilon$. On the set $\mathcal{S}$ the derivative of $V$ coincides for both systems (1) and (3) as it is stated in Proposition 1. The system (3) trajectories obtained for $\sigma=10, \beta=8 / 3, \rho=28$ and $\varepsilon=1$ are shown in Fig. 2. 
Consider the Duffing model:

$$
\left\{\begin{array}{l}
\dot{x}_{1}=x_{2} \\
\dot{x}_{2}=\alpha x_{1}-\beta x_{2}-\gamma x_{1}^{3}
\end{array}\right.
$$

where all parameters $\alpha, \beta$ and $\gamma$ are strictly positive. The system has three equilibria $( \pm \sqrt{\alpha / \gamma}, 0)$, $(0,0)$ (a saddle stationary point at the origin and two stable focuses). The system has a potential function $W\left(x_{1}, x_{2}\right)=0.5\left[\alpha^{2} /(2 \gamma)-\alpha x_{1}^{2}+\gamma / 2 x_{1}^{4}+x_{2}^{2}\right]$, which is zero in the stable steady states $( \pm \sqrt{\alpha / \gamma}, 0)$ and positive otherwise, its time derivative for the Duffing model is not positive: $\dot{W}=-\beta x_{2}^{2}$. The local approximation for $\lambda_{0}=0$ (linearization) allows us to detect that the origin is unstable. For $r_{1}=1, r_{2}=2$ and $d_{0}=1$ the following approximation at $\lambda_{0}=+\infty$ can be obtained:

$$
\mathbf{f}_{0}(\mathbf{x})=\left[\begin{array}{c}
x_{2} \\
-\gamma x_{1}^{3}
\end{array}\right]
$$

for which all trajectories are closed orbits (take a Lyapunov function $U(\mathbf{x})=0.25 x_{1}^{4}+0.5 \gamma^{-1} x_{2}^{2}$, then $\dot{U}=0$ ), thus it does not provide a reliable information about the model global stability. Let us consider the case $0<\lambda_{0}<+\infty$, for this purpose we have to select the set $\mathcal{S}$, around which we are going to compute the system local homogeneous approximation. In this example we selected $\mathcal{S}=\left\{\mathrm{x} \in R^{2}: W\left(x_{1}, x_{2}\right)=q\right\}$ for some $q>0$ big enough in order to ensure that the set $\mathcal{S}$ is connected, i.e. $q>\alpha^{2} /(4 \gamma)$. Take $r_{1}=1, r_{2}=2$, then a straightforward computation shows that

$$
\lambda_{0}(\mathbf{x})=\sqrt{\frac{\sqrt{2} \sqrt{\left[\alpha^{2}-2 q \gamma(2-\gamma)\right] x_{1}^{4}+4 \gamma q-\alpha^{2}}+\sqrt{\gamma} \alpha x_{1}^{2}}{\sqrt{\gamma}\left[(\gamma-2) x_{1}^{4}+2\right]}}
$$

which is well defined on $S_{\mathbf{r}}$, and the local homogeneous approximating dynamics from (2) has the form for $d=d_{0}=0$ :

$$
\mathbf{f}_{0}(\mathbf{x})=\left[\begin{array}{c}
\kappa(\mathbf{x}) x_{2} \\
\alpha \kappa(\mathbf{x})^{-1} x_{1}-\beta x_{2}-\gamma \kappa(\mathbf{x}) x_{1}^{3}
\end{array}\right], \kappa(\mathbf{x})=\sqrt{\frac{\sqrt{2} \sqrt{\left[\alpha^{2}-2 q \gamma(2-\gamma)\right] x_{1}^{4}+\left(4 \gamma q-\alpha^{2}\right)|\mathbf{x}|_{\mathbf{r}}^{4}}+\sqrt{\gamma} \alpha x_{1}^{2}}{\sqrt{\gamma}\left[(\gamma-2) x_{1}^{4}+2|\mathbf{x}|_{\mathbf{r}}^{4}\right]}}
$$

For the approximating dynamics

$$
\dot{W}=L_{\mathbf{f}_{0}} W\left(x_{1}, x_{2}\right)=\alpha\left[\kappa(\mathbf{x})^{-1}-\kappa(\mathbf{x})\right] x_{1} x_{2}-\beta x_{2}^{2}
$$

and $\dot{W}=-\beta x_{2}^{2}$ for all $\mathbf{x} \in \mathcal{S}$, since by construction $\kappa(\mathbf{x})=1$ for $\mathbf{x} \in \mathcal{S}$. Thus the derivative of the function $W$ coincides for both systems on the set of approximation $\mathcal{S}$ as it is stated in Proposition 1 (there the result is given for a positive definite function, but it is also true for a nonnegative definite function). Since the derivative $\dot{W}$ is strictly negative for almost all points on $\mathcal{S}$ and for the points where $x_{2}=0$ the flow directions can be analyzed to justify that the set $\mathcal{X}=\left\{\mathbf{x} \in R^{2}: W\left(x_{1}, x_{2}\right) \leq q\right\}$ is strictly positively invariant, which for the homogeneous approximating system (3) is equivalent to global asymptotic stability at the origin [9]. Since this analysis is correct for all $q>\alpha^{2} /(4 \gamma)$, therefore using this approach we may estimate the set of attraction for Duffing model as $W\left(x_{1}, x_{2}\right) \leq \alpha^{2} /(4 \gamma)$. For $\alpha=\beta=\gamma=1\left(\alpha^{2} /(4 \gamma)=0.25\right)$ the corresponding set $\mathcal{S}$ for $q=0.3$ and the trajectories of the system (3) are shown in Fig. 3. 


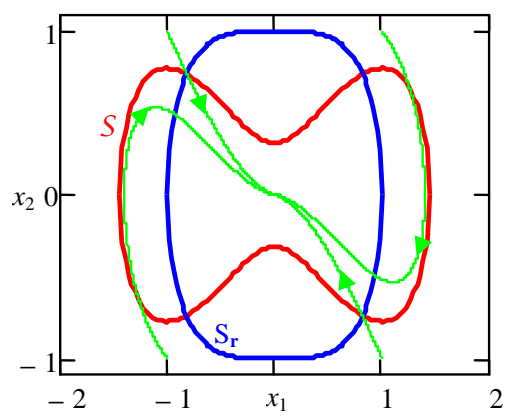

Figure 3. The results of simulation for approximation of the Duffing model

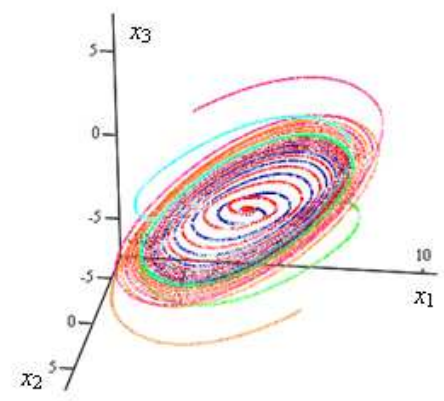

Figure 4. The results of the system (5) simulation

\section{B. Examples for Theorem 6}

Consider the system

$$
\left\{\begin{array}{l}
\dot{x}_{1}=-x_{1}+x_{2}+2 \tanh \left(x_{1}\right) \\
\dot{x}_{2}=-2 x_{1}+x_{3} \\
\dot{x}_{3}=-1.5 x_{1}+2 \tanh \left(x_{1}\right)
\end{array}\right.
$$

where $\mathbf{x}=\left[\begin{array}{lll}x_{1} & x_{2} & x_{3}\end{array}\right]^{T} \in R^{3}$ is the state vector, and the system (5) is in the Lurie form (linear asymptotically stable system closed by a nonlinear feedback). The system (5) has the single equilibrium at the origin, it is homogeneous in the bi-limit, namely $\left(\mathbf{r}_{j}, \lambda_{j}, \mathbf{f}_{j}\right)$-homogeneous with $j=1,2$ and

$$
\begin{gathered}
\mathbf{r}_{1}=\left[\begin{array}{lll}
0.5 & 0.5 & 0.5
\end{array}\right], \lambda_{1}=0, \mathbf{f}_{1}(\mathbf{x})=\mathbf{A}_{1} \mathbf{x}, \mathbf{A}_{1}=\left[\begin{array}{ccc}
1 & 1 & 0 \\
-2 & 0 & 1 \\
0.5 & 0 & 0
\end{array}\right] ; \\
\mathbf{r}_{2}=\left[\begin{array}{lll}
0.5 & 0.5 & 0.5
\end{array}\right], \lambda_{2}=+\infty, \mathbf{f}_{2}(\mathbf{x})=\mathbf{A}_{2} \mathbf{x}, \mathbf{A}_{2}=\left[\begin{array}{ccc}
-1 & 1 & 0 \\
-2 & 0 & 1 \\
-1.5 & 0 & 0
\end{array}\right]
\end{gathered}
$$

with zero degree. All eigenvalues of the matrix $\mathbf{A}_{1}$ have positive real parts, and all eigenvalues of the matrix $\mathbf{A}_{2}$ have negative real parts. Therefore, the conditions of Theorem 6 hold and the system (5) is oscillating, actually the results of simulation presented in Fig. 4 show that it has a stable limit cycle. 


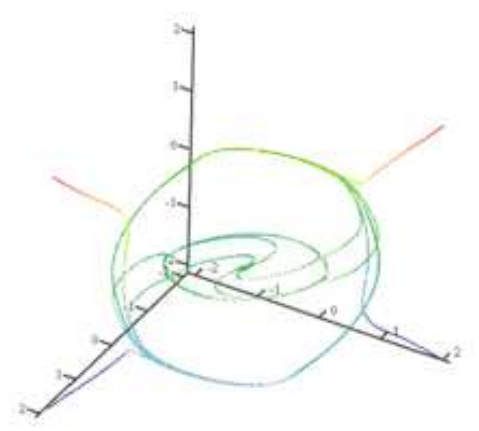

Figure 5. The results of the system (6) simulation

Next, consider the system

$$
\left\{\begin{array}{l}
\dot{x}_{1}=2 x_{1}-\left|0.5 \pi-x_{1}^{2}-x_{2}^{2}-x_{3}^{2}\right| x_{1}^{3}-3\left(x_{1}-x_{2}-x_{3}\right) \cos \left(x_{1}^{2}+x_{2}^{2}+x_{3}^{2}\right) \\
\dot{x}_{2}=x_{2}-\left|0.4 \pi-x_{1}^{2}-x_{2}^{2}-x_{3}^{2}\right| x_{2}^{3}-2\left(2 x_{2}+x_{1}-0.5 x_{3}\right) \cos \left(x_{1}^{2}+x_{2}^{2}+x_{3}^{2}\right) \\
\dot{x}_{3}=-x_{3}-x_{1}-\left|0.6 \pi-x_{1}^{2}-x_{2}^{2}-x_{3}^{3}\right| x_{3}^{3}+\left(2 x_{3}+x_{1}\right) \sin \left(x_{1}^{2}+x_{2}^{2}+x_{3}^{3}\right)
\end{array}\right.
$$

where $\mathbf{x}=\left[\begin{array}{lll}x_{1} & x_{2} & x_{3}\end{array}\right]^{T} \in R^{3}$. The origin is the only equilibrium of the system (6). This system is homogeneous in three limits with

$$
\begin{gathered}
\mathbf{r}_{1}=\left[\begin{array}{lll}
0.5 & 0.5 & 0.5
\end{array}\right], \lambda_{1}=0 \\
\mathbf{r}_{2}=\left[\begin{array}{lll}
0.5 & 0.5 & 0.5
\end{array}\right], \lambda_{2}=+\infty \\
\mathbf{r}_{3}=\left[\begin{array}{lll}
0.5 & 0.5 & 0.5
\end{array}\right], \lambda_{3}=0.5 \pi \\
\mathbf{f}_{1}(\mathbf{x})=\left[\begin{array}{c}
-x_{1}+3 x_{2}+3 x_{3} \\
-3 x_{2}-2 x_{1}+x_{3} \\
-x_{1}-x_{3}
\end{array}\right], \mathbf{f}_{2}(\mathbf{x})=-|\mathbf{x}|^{2}\left[\begin{array}{c}
x_{1}^{3} \\
x_{2}^{3} \\
x_{3}^{3}
\end{array}\right], \mathbf{f}_{3}(\mathbf{x})=\left[\begin{array}{c}
2 x_{1} \\
\left\{1-0.05 \pi^{2}\left[x_{2}^{2} /\left(x_{1}^{2}+x_{2}^{2}+x_{3}^{2}\right)\right]\right\} x_{2} \\
\left\{1-0.05 \pi^{2}\left[x_{3}^{2} /\left(x_{1}^{2}+x_{2}^{2}+x_{3}^{2}\right)\right]\right\} x_{3}
\end{array}\right]
\end{gathered}
$$

with $d_{1}=d_{3}=0$ and $d_{2}=2$. In all three modes the approximating dynamics has the Lyapunov function $V(\mathbf{x})=\mathbf{x}^{T} \mathbf{x}$, and it is easy to verify that the vector fields $\mathbf{f}_{1}, \mathbf{f}_{2}$ are asymptotically stable and $\mathbf{f}_{3}$ is unstable. Thus the conditions of Theorem 6 can be verified twice indicating that the system has two zones with oscillating trajectories. The results of this system simulation presented in Fig. 5 confirm this conclusion, actually the system has two limit cycles (the inner is unstable, and the outer is stable).

Further, consider the system

$$
\left\{\begin{array}{l}
\dot{x}_{1}=-0.5 \operatorname{sign}\left(x_{1}\right) \sqrt{\left|x_{1}\right|}+x_{1}-0.5 x_{1}^{3}-x_{2}-x_{2}^{3} \\
\dot{x}_{2}=-0.5 \operatorname{sign}\left(x_{2}\right) \sqrt{\left|x_{2}\right|}+x_{2}-0.5 x_{2}^{3}+x_{1}+x_{1}^{3}
\end{array},\right.
$$

where $\mathbf{x}=\left[\begin{array}{ll}x_{1} & x_{2}\end{array}\right]^{T} \in R^{2}$. The system (7) has the single equilibrium at the origin, it is homogeneous in two limits and 
additionally the conditions of Corollary 1 are satisfied for the third limit, i.e.

$$
\begin{gathered}
\mathbf{r}_{1}=\left[\begin{array}{ll}
0.5 & 0.5
\end{array}\right], \lambda_{1}=0, \mathbf{f}_{1}(\mathbf{x})=-0.5\left[\begin{array}{c}
\sqrt{\left|x_{1}\right|} \operatorname{sign}\left(x_{1}\right) \\
\sqrt{\left|x_{2}\right|} \operatorname{sign}\left(x_{2}\right)
\end{array}\right] ; \\
\mathbf{r}_{2}=\left[\begin{array}{ll}
0.5 & 0.5
\end{array}\right], \lambda_{2}=+\infty, \mathbf{f}_{2}(\mathbf{x})=\left[\begin{array}{c}
-0.5 x_{1}^{3}-x_{2}^{3} \\
-0.5 x_{2}^{3}+x_{1}^{3}
\end{array}\right],
\end{gathered}
$$

$d_{1}=-0.25$ and $d_{2}=1$ correspondingly. The vector fields $\mathbf{f}_{1}, \mathbf{f}_{2}$ are asymptotically stable with Lyapunov functions

$$
V_{1}(\mathbf{x})=0.5\left(x_{1}^{2}+x_{2}^{2}\right), V_{2}(\mathbf{x})=0.25\left(x_{1}^{4}+x_{2}^{4}\right)
$$

As the third case from Corollary 1 we propose

$$
\mathbf{r}_{3}=\left[\begin{array}{ll}
0.5 & 0.5
\end{array}\right], \lambda_{3}=0.5, \mathbf{f}_{3}(\mathbf{x})=\left[\begin{array}{c}
x_{1}-x_{2} \\
x_{2}+x_{1}
\end{array}\right]
$$

The approximating vector field $\mathbf{f}_{3}$ is unstable with Lyapunov function $V_{3}(\mathbf{x})=0.5\left(x_{1}^{2}+x_{2}^{2}\right)$. Then

$$
D V_{3}(\mathbf{y})\left[\lambda^{-d} \boldsymbol{\Lambda}_{\mathbf{r}_{3}}^{-1} \mathbf{f}\left(\boldsymbol{\Lambda}_{\mathbf{r}_{3}} \mathbf{y}\right)-\mathbf{f}_{3}(\mathbf{y})\right]=-0.5 \lambda^{-0.25}\left(\left|y_{1}\right|^{1.5}+\left|y_{2}\right|^{1.5}\right)+\lambda\left[y_{1}^{3} y_{2}-y_{2}^{3} y_{1}-0.5\left(y_{1}^{4}+y_{2}^{4}\right)\right]
$$

$a=1$ and

$$
\sup _{\mathbf{y} \in S_{\mathbf{r}_{3}}}\left|D V_{3}(\mathbf{y})\left[\lambda^{-d} \boldsymbol{\Lambda}_{\mathbf{r}_{3}}^{-1} \mathbf{f}\left(\boldsymbol{\Lambda}_{\mathbf{r}_{3}} \mathbf{y}\right)-\mathbf{f}_{3}(\mathbf{y})\right]\right|<1
$$

for all $\lambda \in\left(\underline{\lambda}_{3}, \bar{\lambda}_{3}\right)$ where $\underline{\lambda}_{3}=0.16$ and $\bar{\lambda}_{3}=0.55, \underline{\lambda}_{3} \leq \lambda_{3} \leq \bar{\lambda}_{3}$. Thus, according to Corollary 1 , the system (7) is locally unstable into the set $X_{\mathbf{r}_{3}}=\left\{\mathbf{x} \in R^{3}: \underline{\lambda}_{3}<\left|x_{1}\right|^{2}+\left|x_{2}\right|^{2}<\bar{\lambda}_{3}\right\}=\left\{\mathbf{x} \in R^{3}: \sqrt{\lambda_{3}}<|\mathbf{x}|<\sqrt{\bar{\lambda}_{3}}\right\}$. This conclusion can be illustrated by the expression $D V_{3}(\mathbf{x}) \mathbf{f}(\mathbf{x})$, which levels are plotted in Fig. 6, a. The expression represents the time derivative of the Lyapunov function $V_{3}$ calculated for the system (7). Analysis of Fig. 6, a shows that this derivative is strictly positive on some ring around the origin. Consequently, Theorem 6 conditions are satisfied twice for the system (7), and it has two limit cycles as it is confirmed by the results of simulation presented in Fig. 6,b. This example also illustrates the fact that in some cases analysis in bi-limit is not sufficient, and despite of stability of both approximations $\mathbf{f}_{1}(\mathbf{x})$ and $\mathbf{f}_{2}(\mathbf{x})$ the system is not globally asymptotically stable.

Consider a planar system (1) with

$$
\mathbf{f}(\mathbf{x})=\left[\begin{array}{c}
x_{2}+x_{1}\left(\frac{1}{2}-\frac{x_{1}^{2}}{2+x_{2}^{2}}\right) \\
-x_{1}+x_{2}\left(\frac{1}{2}-\frac{x_{2}^{2}}{2+x_{1}^{2}}\right)
\end{array}\right] .
$$

This system has linearization at the origin (local approximation at zero) that is unstable, but this system has no approximation at infinity. Indeed, for $\lambda_{0}=+\infty$ Definition 2 requires that the limit, which defines the local approximation of a system, should be uniform on $S_{\mathbf{r}}$. However, since the numerator and denominator of the functions $\frac{x_{1}^{2}}{2+x_{2}^{2}}$ and $\frac{x_{2}^{2}}{2+x_{1}^{2}}$ contain different variables, 


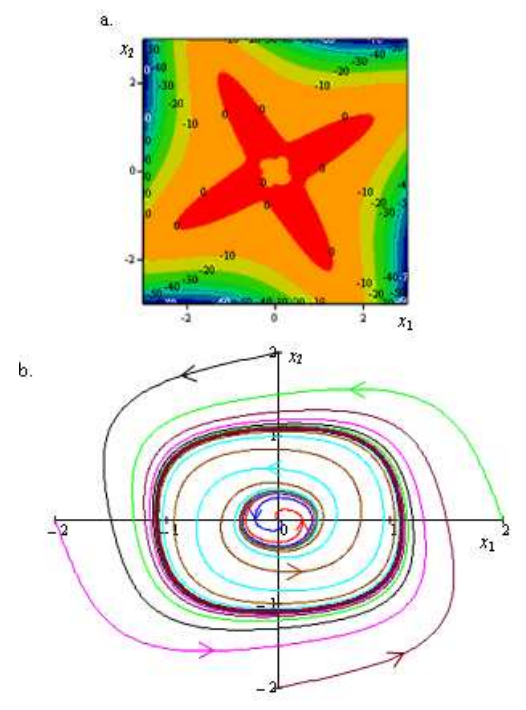

Figure 6. The illustrations for the system (7)

then calculation of a uniform limit is not possible. To illustrate this conclusion consider a particular case $r_{1}=r_{2}=1$, then $\frac{x_{1}^{2}}{2+x_{2}^{2}}=\frac{\lambda^{2} y_{1}^{2}}{2+\lambda^{2} y_{2}^{2}}=\frac{\lambda^{2} y_{1}^{2}}{2+\lambda^{2}\left(1-y_{1}^{2}\right)}$ for $\mathbf{y} \in S_{\mathbf{r}}$ and $\lambda=|\mathbf{x}|$, and for $\lambda \rightarrow+\infty$ there is no uniform limit of $\frac{\lambda^{2} y_{1}^{2}}{2+\lambda^{2}\left(1-y_{1}^{2}\right)}$ for all $y_{1} \in[-1,1]$ (the point $y_{1}= \pm 1$ is singular). From another side, this system has a local homogeneous approximation for any $\lambda_{0} \in(0,+\infty)$, for example, for $r_{1}=r_{2}=1$ and $d=d_{0}=0$ the formula (2) gives:

$$
\mathbf{f}_{0}(\mathbf{x})=\left[\begin{array}{c}
x_{2}+x_{1}\left(\frac{1}{2}-\frac{\lambda_{0}^{2} x_{1}^{2}}{2|\mathbf{x}|^{2}+\lambda_{0}^{2} x_{2}^{2}}\right) \\
-x_{1}+x_{2}\left(\frac{1}{2}-\frac{\lambda_{0}^{2} x_{2}^{2}}{2|\mathbf{x}|^{2}+\lambda_{0}^{2} x_{1}^{2}}\right)
\end{array}\right] .
$$

Take $V(\mathbf{x})=0.5|\mathbf{x}|^{2}$ as a candidate of Lyapunov function for the approximating dynamics (this $V$ is $\mathbf{r}-$ homogeneous), its time derivative for $\mathbf{f}_{0}$ has the form:

$$
\dot{V}\left(\mathbf{x}, \lambda_{0}\right)=x_{1}^{2}\left(\frac{1}{2}-\frac{\lambda_{0}^{2} x_{1}^{2}}{2|\mathbf{x}|^{2}+\lambda_{0}^{2} x_{2}^{2}}\right)+x_{2}^{2}\left(\frac{1}{2}-\frac{\lambda_{0}^{2} x_{2}^{2}}{2|\mathbf{x}|^{2}+\lambda_{0}^{2} x_{1}^{2}}\right) .
$$

Since $V$ and $\mathbf{f}_{0}$ are $\mathbf{r}$-homogeneous, then sign definiteness of this function can be analyzed on the sphere $S_{\mathbf{r}}$ only, i.e. for the function $v\left(x_{1}, \lambda_{0}\right)=\left.\dot{V}\left(\mathbf{x}, \lambda_{0}\right)\right|_{|\mathbf{x}|=1, x_{2}^{2}=1-x_{1}^{2}}$ with $x_{1} \in[-1,1]$. The partial derivative of $v$ with respect to $x_{1}$ has three roots: $\pm \frac{1}{\sqrt{2}}$ and 0 . A simple analysis shows that $v\left(\lambda_{0}, \pm \frac{1}{\sqrt{2}}\right)=\frac{4-\lambda_{0}^{2}}{2\left(4+\lambda_{0}^{2}\right)}$ is the maximum and $v\left(\lambda_{0}, 0\right)=v\left(\lambda_{0}, \pm 1\right)=\frac{1-\lambda_{0}^{2}}{2}$ is the minimum of $v$ on $[-1,1]$. Therefore, $\dot{V}\left(\mathbf{x}, \lambda_{0}\right)<0$ if $\lambda_{0}>2$ and $\dot{V}\left(\mathbf{x}, \lambda_{0}\right)>0$ if $\lambda_{0}<1$ for all $\mathbf{x} \in S_{\mathbf{r}}$, which due to the homogeneity arguments implies asymptotic stability at the origin of $\mathbf{f}_{0}$ for $2<\lambda_{0}<+\infty$ and instability for $0<\lambda_{0}<1$. According to Proposition 1, the original system (1) has to inherit this properties, and $V$ is the corresponding Lyapunov function for its stability/instability test. Since the system has the single equilibrium point at the origin, then according to Theorem 6 it is oscillating (as a planar system, it has a limit cycle). The trajectories of this system and the obtained estimates on location of the limit cycle are shown in Fig. 7.

Finally, consider the system

$$
\left\{\begin{array}{l}
\dot{x}_{1}=-x_{1}+1.5 x_{2}-0.3 x_{1}^{3}+x_{1} x_{2}^{2} \\
\dot{x}_{2}=-x_{1}+2 x_{2}-0.5 x_{2}^{3}-x_{2} x_{1}^{2}
\end{array}\right.
$$




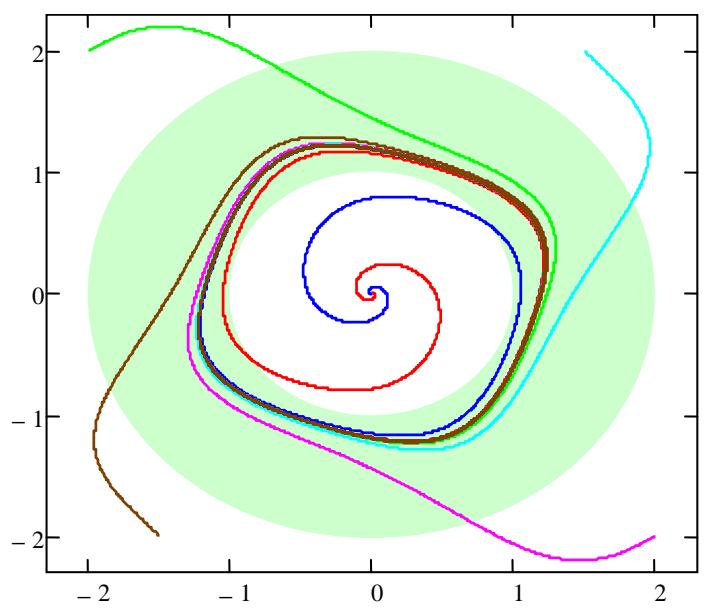

Figure 7. The results of simulation for a system without approximation at infinity

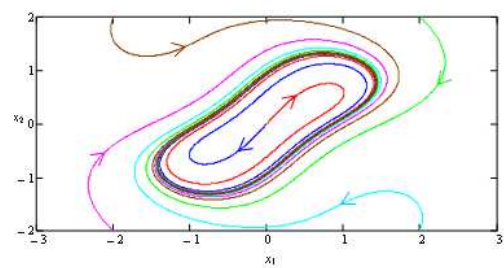

Figure 8. The trajectories of the system (9)

where $\mathbf{x}=\left[x_{1} x_{2}\right]^{T} \in R^{2}$. The system (9) has the single equilibrium at the origin, it is homogeneous in two limits:

$$
\begin{aligned}
& \mathbf{r}_{1}=\left[\begin{array}{ll}
0.5 & 0.5
\end{array}\right], \lambda_{1}=0, \mathbf{f}_{1}(\mathbf{x})=\mathbf{A x}, \mathbf{A}=\left[\begin{array}{cc}
-1 & 1.5 \\
-1 & 2
\end{array}\right] ; \\
& \mathbf{r}_{2}=\left[\begin{array}{ll}
0.5 & 0.5
\end{array}\right], \lambda_{2}=+\infty, \mathbf{f}_{2}(\mathbf{x})=\left[\begin{array}{c}
-0.3 x_{1}^{3}+x_{1} x_{2}^{2} \\
-0.5 x_{2}^{3}-x_{1}^{2} x_{2}
\end{array}\right],
\end{aligned}
$$

$d_{1}=0$ and $d_{2}=1$ respectively. The vector field $\mathbf{f}_{1}$ is linear and unstable with the Chetaev function

$$
V_{1}(\mathbf{x})=0.5\left(x_{1}^{2}+x_{2}^{2}\right)
$$

and the cone $K=\left\{\mathbf{x} \in R^{2}: x_{2} \geq\left|x_{1}\right|\right\}$ (the case of Proposition 3 ), $\mathbf{f}_{2}$ is asymptotically stable with the Lyapunov function

$$
V_{2}(\mathbf{x})=0.25\left(x_{1}^{2}+x_{2}^{2}\right)
$$

Therefore, applying arguments similar to ones used in Theorem 6 we can substantiate existence of a limit cycle (the trajectories are globally bounded and the single equilibrium is unstable, therefore, there exists a compact set attracting almost all trajectories, the system is oscillating in the sense of Yakubovich that for planar systems is equivalent to a limit cycle existence). The trajectories of the system are plotted in Fig. 8. 


\section{CONCLUSiON}

The notion of local homogeneity is introduced. To use this property it is required to find an approximating dynamics (3) (the formulas (2) are given providing examples of approximating homogeneous functions in a common case). To analyze stability or instability of the approximating dynamics (3) the Lyapunov or Chetaev function methods can be used, the first order approximation (4) at the origin can also be applied. The result of this work claims that the original system (1) inherits locally stability or instability of the approximating dynamics (3), as well as it locally accedes to the corresponding Lyapunov or Chetaev functions. The necessary and sufficient conditions of instability for homogeneous systems in terms of Lyapunov and Chetaev functions are established.

The paper presents conditions of oscillation existence for systems homogeneous in the multi-limit. The conditions allows the presence of multiple oscillating modes to be detected for homogeneous in several limits vector fields (that is a development with respect to [24], [25]). Efficiency of the proposed conditions is demonstrated on several examples. To detect oscillations we have to divide the state space on subsets where the system is stable or unstable. For a generic nonlinear system there exists only one approach to prove stability/instability, it is the second Lyapunov method. The application of this method is difficult, since there is no technique for the Lyapunov (Chetaev) function choice in a particular example. The proposed approach, based on homogeneity in the multi-limit, allows us to decompose this complex unstructured problem into several simpler ones.

The main differences of the local homogeneity for $0<\lambda_{0}<+\infty$ with respect to introduced previously approximations for $\lambda_{0}=0$ and $\lambda_{0}=+\infty$ are that the former ones exist for any continuous system (1) (expressions of the approximating functions $\mathbf{f}_{0}$ at infinity or zero may be impossible to compute, see example (8) in Section IV), and that approximations for $0<\lambda_{0}<+\infty$ provide additional local information on stability/instability of nonlinear systems that can be useful in some applications, for detection of oscillations for instance.

\section{REFERENCES}

[1] V. Andrieu, L. Praly, and A. Astolfi, "Homogeneous approximation, recursive observer design, and output feedback," SIAM J. Control Optimization, vol. 47 , no. 4 , pp. $1814-1850,2008$.

[2] V. Zubov, "On ordinary differential equations with generalized homogeneous right-hand sides," Izvestiya vuzov, Matematika, vol. 1, no. 2, pp. 80-88, 1958.

[3] L. Rothschild and E. Stein, "Hypoelliptic differential operators and nilpotent groups," Acta Mathematica, vol. 137, pp. 247-320, 1976.

[4] A. Bacciotti and L. Rosier, Liapunov Functions and Stability in Control Theory, vol. 267 of Lecture Notes in Control and Inform. Sci. Berlin: Springer, 2001.

[5] H. Hermes, Homogeneous coordinates and continuous asymptotically stabilizing feedback controls, vol. 109 of Differential Equations: Stability and Control, pp. 249-260. Marcel Dekker, 1991.

[6] Y. Hong, "Finite-time stabilization and stabilizability of a class of controllable systems," Systems\&Control Lett., vol. 46, pp. 231-236, 2002.

[7] L. Rosier, "Homogeneous lyapunov function for homogeneous continuous vector field," Systems\&Control Lett., vol. 19, pp. 467-473, 1992.

[8] H. Hermes, "Nilpotent and high-order approximations of vector field systems," SIAM Review, vol. 33, no. 2, pp. $238-264,1991$.

[9] S. Bhat and D. Bernstein, "Geometric homogeneity with applications to finite-time stability," Mathematics of Control, Signals and Systems, vol. 17, pp. 101-127, 2005.

[10] L. Grüne, "Homogeneous state feedback stabilization of homogeneous systems," SIAM J. Control Optimization, vol. 38, no. 4, pp. 1288-1314, 2000.

[11] M. Kawski, Homogeneous feedback stabilization, vol. 7 of Progress in systems and control theory: New trends in systems theory. Birkhuser, 1991. 
[12] E. Moulay and W. Perruquetti, "Finite time stability and stabilization of a class of continuous systems," J. Mathematical Analysis Applications, vol. 323, no. 2, pp. 1430-1443, 2006.

[13] L. Praly, "Generalized weighted homogeneity and state dependent time scale for linear controllable systems," in Proc. IEEE CDC 1997, (San Diego), pp. 4342-4347, IEEE, 1997.

[14] R. Sepulchre and D. Aeyels, "Stabilizability does not imply homogeneous stabilizability for controllable systems," SIAM J. Control Optimization, vol. 34, no. 5, pp. 1798-1813, 1996.

[15] T. Menard, E. Moulay, and W. Perruquetti, “A global high-gain finite-time observer," IEEE Trans. Autom. Control, vol. 55, no. 6, pp. 1500-1506, 2010.

[16] N. Chetaev, The Stability of Motion. New York: Pergamon Press, 1961. (English translation).

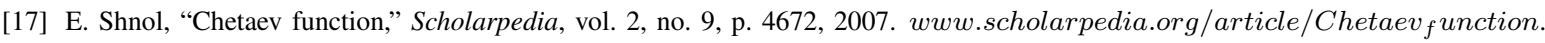

[18] A. Fradkov and A. Pogromsky, Introduction to oscillations and chaos. Singapore: World Scientific, 1998.

[19] G. Leonov, I. Burkin, and A. Shepelyavyi, Frequency Methods in Oscillation Theory. Dordrecht: Kluwer, 1995. in Russian: 1992.

[20] S. Martinez, J. Cortes, and F. Bullo, “Analysis and design of oscillatory control systems," IEEE Trans. Autom. Control, vol. 48, no. 7, pp. 1164-1177, 2003.

[21] F. D. Meglio, G.-O. Kaasa, and N. Petit, "A first principle model for multiphase slugging flow in vertical risers," in Proc. 48th IEEE Conf. on Decision and Control, (Shanghai), pp. 8244-8251, IEEE, 2009.

[22] V. Yakubovich, "Frequency oscillations conditions in nonlinear systems with stationary single nonlinearity," Siberian Math. J., vol. 14, no. 2, 1973.

[23] V. Yakubovich, “Oscillations in systems with discontinuous and hysteresis nonlinearities," Automation and Remote Control, vol. 12, 1975.

[24] V. Yakubovich and E. Tomberg, "Conditions for self-induced oscillations in nonlinear systems," Siberian Math. J., vol. 30, pp. 641-653, 1989.

[25] D. Efimov and A. Fradkov, "Oscillatority of nonlinear systems with static feedback," SIAM J. Control Optimization, vol. 48, no. 2, pp. 618-640, 2009.

[26] H. Khalil, Nonlinear Systems. Upper Saddle River, New Jersey: Prentice Hall, 3rd ed., 2002.

[27] Y. Lin, E. Sontag, and Y. Wang, "A smooth converse lyapunov theorem for robust stability," SIAM J. Control Optimization, vol. 34, no. 1, pp. 124-160, 1996.

[28] E. Bernuau, A. Polyakov, D. Efimov, and W. Perruquetti, "On extension of homogeneity notion for differential inclusions," in Proc. European Control Conference (ECC), 2013.

[29] D. Efimov and A. Fradkov, "Yakubovichÿs oscillatority of circadian oscillations models," Mathematical Biosciences, vol. 216, pp. 187-191, 2008.

[30] L. Khazin and E. Shnol, Stability of Critical Equilibrium States. Manchester University Press, 1991.

[31] V. Zubov, Methods of A. M. Lyapunov and their application. Groningem: P. Noordhoff, 1964.

[32] N. Krasovskii, Stability of motion: Applications of Lyapunov's second method to differential systems and equations with delay. Stanford University Press, 1963.

[33] R. Rajamani, “Observers for lipschitz nonlinear systems,” IEEE Trans. Autom. Control, vol. 43, pp. 397-401, 1998.

[34] F. Zhu and Z. Han, “A note on observers for lipschitz nonlinear systems,” IEEE Trans. Autom. Control, vol. 47, pp. 1751-1754, 2002.

[35] M.-S. Chen and C.-C. Chen, "Robust nonlinear observer for lipschitz nonlinear systems subject to disturbances," IEEE Trans. Autom. Control, vol. 52, pp. 2365-2370, 2007.

[36] M. Arcak and P. Kokotović, "Nonlinear observers: A circle criterion design and robustness analysis," Automatica, vol. 37, pp. 1923-1930, 2001.

[37] D. Efimov and W. Perruquetti, “Oscillating system design applying universal formula for control," in Proc. 50th IEEE CDC-ECC 2011, (Orlando, FL), pp. 1747-1752, 2011.

[38] N. P. Bhatia and G. Szegö, Dynamical systems: stability theory and applications. Springer-Verlag, 1967.

\section{APPENDIX}

\section{Proof of Lemma 1}

The sufficient part is clear (appearance of the corresponding Lyapunov function implies instability [16]). The necessary existence of the locally Lipschitz continuous Lyapunov function $V$ with the properties a) and b) has been proven in [25]. To prove existence of a continuously differentiable and homogeneous Lyapunov function simply note that the time reversing in the system (1) 
implies that the system $\dot{\mathbf{x}}=-\mathbf{f}(\mathbf{x})$ with the trajectories $\mathbf{x}\left(-t, \mathbf{x}_{0}\right)$ is locally asymptotically stable. This fact according to Theorem 1 implies existence of a homogeneous and continuously differentiable Lyapunov function satisfying a), b) and c).

\section{Proof of Lemma 2}

According to the lemma conditions, under constraints $0<\left|\mathbf{x}_{0}\right|<\delta$ and $\mathbf{x}_{0} \in K$ one can take initial conditions as close as possible to the origin such that the corresponding trajectory leaves the set $B_{\delta}$ in the finite time $T_{\mathbf{x}_{0}}$ through the cone $K$ basement (the lateral borders $\mathrm{lb}(K)$ are all points of entrance). Then, the sufficient part $((i i) \Rightarrow(i))$ follows by the Chetaev theorem: if there exist the defined function $V$ with $B_{\delta}$, then the system (1) is unstable [16].

To prove the necessary part $((i) \Rightarrow(i i))$, note that by conditions for all $\mathbf{x}_{0} \in B_{\delta}$ there exists $T_{\mathbf{x}_{0}} \in R_{+}$such that $\mathbf{x}\left(t, \mathbf{x}_{0}\right) \notin B_{\delta}$ for $t \geq T_{\mathbf{x}_{0}}$. Define

$$
v\left(\mathbf{x}_{0}\right)=\inf _{0 \leq t \leq T_{\mathbf{x}_{0}}}\left|\mathbf{x}\left(t, \mathbf{x}_{0}\right)\right|
$$

by construction $\eta\left(\left|\mathbf{x}_{0}\right|\right) \leq v\left(\mathbf{x}_{0}\right) \leq\left|\mathbf{x}_{0}\right|$, where $\eta(s)=s(1+s)^{-1} \inf _{s \leq|\mathbf{x}| \leq \delta, \mathbf{x} \in K} v(\mathbf{x}), \eta \in \mathcal{K}$ and $v(0)=0$. To analyze continuity property of the function $v$ consider

$$
\left|v\left(\mathbf{x}_{1}\right)-v\left(\mathbf{x}_{2}\right)\right|=\left|\inf _{0 \leq t \leq T_{\mathbf{x}_{1}}}\right| \mathbf{x}\left(t, \mathbf{x}_{1}\right)\left|-\inf _{0 \leq t \leq T_{\mathbf{x}_{2}}}\right| \mathbf{x}\left(t, \mathbf{x}_{2}\right)|| \leq \sup _{0 \leq t \leq T}|| \mathbf{x}\left(t, \mathbf{x}_{1}\right)|-| \mathbf{x}\left(t, \mathbf{x}_{2}\right)||
$$

where $T=\max \left\{T_{\mathbf{x}_{1}}, T_{\mathbf{x}_{2}}\right\}$. Due to Lipschitz continuity of the system (1) solutions on any compact set of initial conditions $\mathcal{D} \subset B_{\delta}$ and time $0 \leq T<+\infty$ there exists $L \in R_{+}$such that $\left|\mathbf{x}\left(t, \mathbf{x}_{1}\right)-\mathbf{x}\left(t, \mathbf{x}_{2}\right)\right| \leq L\left|\mathbf{x}_{1}-\mathbf{x}_{2}\right|$, for all $0 \leq t \leq T$ and any $\mathbf{x}_{1}, \mathbf{x}_{2} \in \mathcal{D}$. For all $0<\delta^{\prime}<\delta$ there exists $T_{\delta^{\prime}}=\sup _{\mathbf{x}_{0} \in B_{\delta} \backslash B_{\delta^{\prime}}} T_{\mathbf{x}_{0}}$ with the properties $T_{\delta^{\prime}}<+\infty$ and $T_{\delta^{\prime}} \rightarrow+\infty$ for $\delta^{\prime} \rightarrow 0$, then

$$
\left|v\left(\mathbf{x}_{1}\right)-v\left(\mathbf{x}_{2}\right)\right| \leq \sup _{0 \leq t \leq T_{\delta^{\prime}}}|| \mathbf{x}\left(t, \mathbf{x}_{1}\right)|-| \mathbf{x}\left(t, \mathbf{x}_{2}\right)|| \leq L\left|\mathbf{x}_{1}-\mathbf{x}_{2}\right|
$$

for all $\mathbf{x}_{1}, \mathbf{x}_{2} \in B_{\delta} \backslash B_{\delta^{\prime}}$ and the function $v$ is locally Lipschitz continuous on the set $B_{\delta} \backslash B_{\delta^{\prime}}$ for any fixed $0<\delta^{\prime}<\delta$. Therefore, it is locally Lipschitz continuous on $B_{\delta}$ and continuous on $B_{\delta} \cup\{0\}$. The function $v$ is not decreasing on trajectories of the system (1) for any $\mathbf{x}_{0} \in B_{\delta}$ :

$$
v\left(\mathbf{x}\left(t, \mathbf{x}_{0}\right)\right)=\inf _{0 \leq \tau \leq T_{\mathbf{x}\left(t, \mathbf{x}_{0}\right)}}\left|\mathbf{x}\left(\tau, \mathbf{x}\left(t, \mathbf{x}_{0}\right)\right)\right|=\inf _{t \leq \tau \leq T_{\mathbf{x}_{0}}}\left|\mathbf{x}\left(\tau, \mathbf{x}_{0}\right)\right| \geq \inf _{0 \leq \tau \leq T_{\mathbf{x}_{0}}}\left|\mathbf{x}\left(\tau, \mathbf{x}_{0}\right)\right|=v\left(\mathbf{x}_{0}\right)
$$

To design a strictly increasing function, let us define a new function for all $\mathbf{x}_{0} \in B_{\delta}$ :

$$
V\left(\mathbf{x}_{0}\right)=\inf _{0 \leq t \leq T_{\mathbf{x}_{0}}}\left\{v\left(\mathbf{x}\left(t, \mathbf{x}_{0}\right)\right) k(t)\right\}
$$

where $k: R_{+} \rightarrow R_{+}$is a continuously differentiable function with the properties $0<\kappa_{1} \leq k(t) \leq \kappa_{2}<+\infty$ and $\dot{k}(t) \leq-\kappa_{3}(t)<0$ for all $t \geq 0$ [25], [27]. An example of such a function is as follows:

$$
k(t)=\kappa_{1}+\left(\kappa_{2}-\kappa_{1}\right) e^{-\omega t}, \dot{k}(t)=\omega\left(\kappa_{1}-\kappa_{2}\right) e^{-\omega t}, \omega>0 .
$$


The function $V$ has bounds $\kappa_{1} \eta\left(\left|\mathbf{x}_{0}\right|\right) \leq V\left(\mathbf{x}_{0}\right) \leq \kappa_{2}\left|\mathbf{x}_{0}\right|$ and $V(0)=0$. Next, for all $\mathbf{x}_{1}, \mathbf{x}_{2} \in B_{\delta}$

$$
\begin{aligned}
\left|V\left(\mathbf{x}_{1}\right)-V\left(\mathbf{x}_{2}\right)\right| & =\left|\inf _{0 \leq t \leq T_{\mathbf{x}_{1}}}\left\{v\left(\mathbf{x}\left(t, \mathbf{x}_{1}\right)\right) k(t)\right\}-\inf _{0 \leq t \leq T_{\mathbf{x}_{2}}}\left\{v\left(\mathbf{x}\left(t, \mathbf{x}_{2}\right)\right) k(t)\right\}\right| \\
& =\left|\inf _{0 \leq t \leq T}\left\{v\left(\mathbf{x}\left(t, \mathbf{x}_{1}\right)\right) k(t)\right\}-\inf _{0 \leq t \leq T}\left\{v\left(\mathbf{x}\left(t, \mathbf{x}_{2}\right)\right) k(t)\right\}\right| \\
& \leq \sup _{0 \leq t \leq T}\left|k(t)\left[v\left(\mathbf{x}\left(t, \mathbf{x}_{1}\right)\right)-v\left(\mathbf{x}\left(t, \mathbf{x}_{2}\right)\right)\right]\right| \\
& \leq \kappa_{2} \sup _{0 \leq t \leq T}\left|v\left(\mathbf{x}\left(t, \mathbf{x}_{1}\right)\right)-v\left(\mathbf{x}\left(t, \mathbf{x}_{2}\right)\right)\right|
\end{aligned}
$$

where $T=\max \left\{T_{\mathbf{x}_{1}}, T_{\mathbf{x}_{2}}\right\}$. For all $0<\delta^{\prime}<\delta$ there exists $T_{\delta^{\prime}}=\sup _{\mathbf{x}_{0} \in B_{\delta} \backslash B_{\delta^{\prime}}} T_{\mathbf{x}_{0}}<+\infty$ and

$$
\left|V\left(\mathbf{x}_{1}\right)-V\left(\mathbf{x}_{2}\right)\right| \leq \kappa_{2} \sup _{0 \leq t \leq T_{\delta^{\prime}}}\left|v\left(\mathbf{x}\left(t, \mathbf{x}_{1}\right)\right)-v\left(\mathbf{x}\left(t, \mathbf{x}_{2}\right)\right)\right| \leq \kappa_{2} L\left|\mathbf{x}\left(t, \mathbf{x}_{1}\right)-\mathbf{x}\left(t, \mathbf{x}_{2}\right)\right| \leq \kappa_{2} L^{2}\left|\mathbf{x}_{1}-\mathbf{x}_{2}\right|
$$

for all $\mathbf{x}_{1}, \mathbf{x}_{2} \in B_{\delta} \backslash B_{\delta^{\prime}}$. Then the function $V$ is locally Lipschitz continuous on the set $B_{\delta}$ and continuous onto $B_{\delta} \cup\{0\}$. It is strictly increasing for any $\mathbf{x}_{0} \in B_{\delta}$ :

$$
\begin{aligned}
V\left(\mathbf{x}\left(t, \mathbf{x}_{0}\right)\right) & =\inf _{0 \leq \tau \leq T_{\mathbf{x}\left(t, \mathbf{x}_{0}\right)}}\left\{v\left(\mathbf{x}\left[\tau, \mathbf{x}\left(t, \mathbf{x}_{0}\right)\right]\right) k(\tau)\right\} \\
& \geq \inf _{t \leq \tau \leq T_{\mathbf{x}_{0}}}\left\{v\left(\mathbf{x}\left[\tau, \mathbf{x}_{0}\right]\right) k(\tau-t)\right\} \\
& >\inf _{0 \leq \tau \leq T_{\mathbf{x}_{0}}}\left\{v\left(\mathbf{x}\left[\tau, \mathbf{x}_{0}\right]\right) k(\tau)\right\}=V\left(\mathbf{x}_{0}\right) .
\end{aligned}
$$

Since $V(t)$ is identically zero at the origin, then

$$
L_{\mathbf{f}\left(\mathbf{x}_{0}\right)} V\left(\mathbf{x}_{0}\right)=\lim _{h \rightarrow 0} h^{-1}\left[V\left(\mathbf{x}\left(h, \mathbf{x}_{0}\right)\right)-V\left(\mathbf{x}_{0}\right)\right]>0
$$

for a.e. $\mathbf{x}_{0} \in B_{\delta}$. By definition

$$
\begin{aligned}
V\left(\mathbf{x}\left(h, \mathbf{x}_{0}\right)\right) & =\inf _{0 \leq t \leq T_{\mathbf{x}\left(h, \mathbf{x}_{0}\right)}}\left\{v\left(\mathbf{x}\left[t, \mathbf{x}\left(h, \mathbf{x}_{0}\right)\right]\right) k(t)\right\} \\
& =\inf _{h \leq t \leq T_{\mathbf{x}_{0}}}\left\{v\left(\mathbf{x}\left[t, \mathbf{x}_{0}\right]\right) k(t-h)\right\}=\inf _{h \leq t \leq T_{\mathbf{x}_{0}}}\left\{v\left(\mathbf{x}\left[t, \mathbf{x}_{0}\right]\right) k(t) k(t)^{-1} k(t-h)\right\} \\
& \geq \inf _{h \leq t \leq T_{\mathbf{x}_{0}}}\left\{v\left(\mathbf{x}\left[t, \mathbf{x}_{0}\right]\right) k(t)\right\} \inf _{h \leq t \leq T_{\mathbf{x}_{0}}}\left\{k(t)^{-1} k(t-h)\right\} \\
& \geq V\left(\mathbf{x}_{0}\right) \inf _{h \leq t \leq T_{\mathbf{x}_{0}}}\left\{k(t)^{-1} k(t-h)\right\} .
\end{aligned}
$$

Finally

$$
\begin{aligned}
\lim _{h \rightarrow 0} h^{-1}\left[V\left(\mathbf{x}\left(h, \mathbf{x}_{0}\right)\right)-V\left(\mathbf{x}_{0}\right)\right] & \geq \lim _{h \rightarrow 0} h^{-1}\left[V\left(\mathbf{x}_{0}\right) \inf _{h \leq t \leq T_{\mathbf{x}_{0}}}\left\{k(t)^{-1} k(t-h)\right\}-V\left(\mathbf{x}_{0}\right)\right] \\
& =V\left(\mathbf{x}_{0}\right) \lim _{h \rightarrow 0} h^{-1}\left[\inf _{h \leq t \leq T_{\mathbf{x}_{0}}}\left\{k(t)^{-1} k(t-h)\right\}-1\right] \\
& =V\left(\mathbf{x}_{0}\right) \lim _{h \rightarrow 0} h^{-1} \inf _{h \leq t \leq T_{\mathbf{x}_{0}}} k(t)^{-1}\{k(t-h)-k(t)\} \\
& \geq V\left(\mathbf{x}_{0}\right) \inf _{0 \leq t \leq T_{\mathbf{x}_{0}}} k(t)^{-1} \lim _{h \rightarrow 0} h^{-1}\{k(t-h)-k(t)\} \\
& =V\left(\mathbf{x}_{0}\right) \inf _{0 \leq t \leq T_{\mathbf{x}_{0}}}\left\{-k(t)^{-1} \dot{k}(t)\right\} \geq \kappa_{2}^{-1} \kappa_{3}\left(T_{\mathbf{x}_{0}}\right) V\left(\mathbf{x}_{0}\right) .
\end{aligned}
$$


We substantiate the inequality

$$
L_{\mathbf{f}(\mathbf{x})} V(\mathbf{x}) \geq \alpha_{3}(V(\mathbf{x})), \alpha_{3}(s)=\kappa_{2}^{-1} s \inf _{V(\mathbf{x}) \geq s \wedge \mathbf{x} \in B_{\delta}} \kappa_{3}\left(T_{\mathbf{x}}\right)
$$

for a.e. $\mathbf{x} \in B_{\delta}$ (the inequality is additionally valid at $\mathbf{x}=0$ ), where $\alpha_{1}(s)=\kappa_{1} \eta(s), \alpha_{2}(s)=\kappa_{2} s$. Existence of a continuously differentiable function $V$ follows next by standard smoothing arguments [27].

Let $\mathbf{f}: R^{n} \rightarrow R^{n}$ be $\mathbf{r}$-homogeneous with degree $d$. In this case it is worth to reformulate all conditions of the theorem using the homogeneous norm $|\cdot|_{\mathbf{r}}$ instead of the Euclidean norm $|\cdot|$, in this case the value of $\delta>0$ can be chosen arbitrary, i.e. $B_{\delta}=K$. In addition, in this case $K$ should be invariant with respect to dilation transformation $\Lambda_{\mathbf{r}}$. Let $V: R^{n} \rightarrow R_{+}$be the continuously differentiable Chetaev function obtained on the previous step with this new norm. As in [7] define

$$
W(\mathbf{x})=\left\{\begin{array}{l}
\int_{0}^{+\infty} a\left[V\left(\Lambda_{\mathbf{r}} \mathbf{x}\right)\right] / \lambda^{k+1} d \lambda \text { if } \mathbf{x} \in K \backslash\{0\} \\
0 \text { if } \mathbf{x}=0
\end{array}\right.
$$

where $a: R_{+} \rightarrow R_{+}$is a smooth function with the properties:

(i) $\quad a(s)=0$ for all $s \leq 1$;

(ii) $\quad a^{\prime}(s)>0$ for all $1<s<2, a^{\prime}(s)=0$ for all $s \geq 2$.

By this construction the function $W$ is positive definite and continuously differentiable. A direct computation shows that this function is $\mathbf{r}$-homogeneous, i.e. for $\mathbf{x} \neq 0$

$$
\begin{aligned}
& W\left(\Lambda_{\mathbf{r}} \mathbf{x}\right)=\int_{0}^{+\infty} a\left[V\left(\Lambda_{\mathbf{r}} \Lambda_{\mathbf{r}}^{\prime} \mathbf{x}\right)\right] / \lambda^{\prime k+1} d \lambda^{\prime} \\
&=\lambda^{k} \quad \int_{0}^{+\infty} a\left[V\left(\Lambda_{\mathbf{r}}^{\prime \prime} \mathbf{x}\right)\right] / \lambda^{\prime \prime k+1} d \lambda^{\prime \prime}=\lambda^{k} W(\mathbf{x})
\end{aligned}
$$

where the change of variables $\lambda^{\prime \prime}=\lambda \lambda^{\prime}$ has been used on the last step. Then

$$
\alpha_{1}\left(|\mathbf{x}|_{\mathbf{r}}\right)=\min _{\mathbf{y} \in S_{\mathbf{r}}}\{W(\mathbf{y})\}|\mathbf{x}|_{\mathbf{r}}^{k}, \alpha_{2}\left(|\mathbf{x}|_{\mathbf{r}}\right)=\max _{\mathbf{y} \in S_{\mathbf{r}}}\{W(\mathbf{y})\}|\mathbf{x}|_{\mathbf{r}}^{k}
$$

Its time derivative for the system (1) is a continuous function and it has the following form for all $\mathbf{x} \in K \backslash\{0\}$ :

$$
\begin{aligned}
\dot{W}(\mathbf{x}) & =\int_{0}^{+\infty} a^{\prime}\left[V\left(\Lambda_{\mathbf{r}} \mathbf{x}\right)\right] D V\left(\Lambda_{\mathbf{r}} \mathbf{x}\right) \Lambda_{\mathbf{r}} \mathbf{f}(\mathbf{x}) / \lambda^{k+1} d \lambda \\
& =\int_{0}^{+\infty} a^{\prime}\left[V\left(\Lambda_{\mathbf{r}} \mathbf{x}\right)\right] D V\left(\Lambda_{\mathbf{r}} \mathbf{x}\right) \mathbf{f}\left(\Lambda_{\mathbf{r}} \mathbf{x}\right) / \lambda^{k+d+1} d \lambda \\
& =\int_{\underline{\lambda}(\mathbf{x})}^{\bar{\lambda}(\mathbf{x})} a^{\prime}\left[V\left(\Lambda_{\mathbf{r}} \mathbf{x}\right)\right] D V\left(\Lambda_{\mathbf{r}} \mathbf{x}\right) \mathbf{f}\left(\Lambda_{\mathbf{r}} \mathbf{x}\right) / \lambda^{k+d+1} d \lambda
\end{aligned}
$$

where $\underline{\lambda}(\mathbf{x})=\arg \min _{\lambda \in R_{+}}\left\{V\left(\Lambda_{\mathbf{r}} \mathbf{x}\right)=1\right\}$ and $\bar{\lambda}(\mathbf{x})=\arg \max _{\lambda \in R_{+}}\left\{V\left(\Lambda_{\mathbf{r}} \mathbf{x}\right)=2\right\}$. Owing the functions $a$ and $V$ properties we have $a^{\prime}\left[V\left(\Lambda_{\mathbf{r}} \mathbf{x}\right)\right] D V\left(\Lambda_{\mathbf{r}} \mathbf{x}\right) \mathbf{f}\left(\Lambda_{\mathbf{r}} \mathbf{x}\right) / \lambda^{k+d+1}>0$ for all $\mathbf{x} \in K \backslash\{0\}$ and $\lambda \in[\underline{\lambda}(\mathbf{x}), \bar{\lambda}(\mathbf{x})]\left(a^{\prime}\left[V\left(\Lambda_{\mathbf{r}} \mathbf{x}\right)\right]=0\right.$ for other $\left.\lambda\right)$. Thus $\dot{W}(\mathbf{x})>0$ for all $\mathbf{x} \in K \backslash\{0\}$ and $\dot{W}(0)=0$, then $\alpha_{3}(s)=\widetilde{\alpha}_{3} \circ \alpha_{2}^{-1}(s)$ where

$$
\widetilde{\alpha}_{3}(s)=\inf _{s<|\mathbf{x}|_{\mathbf{r}}, \mathbf{x} \in K} \int_{\underline{\lambda}(\mathbf{x})}^{\bar{\lambda}(\mathbf{x})} a^{\prime}\left[V\left(\Lambda_{\mathbf{r}} \mathbf{x}\right)\right] D V\left(\Lambda_{\mathbf{r}} \mathbf{x}\right) \mathbf{f}\left(\Lambda_{\mathbf{r}} \mathbf{x}\right) / \lambda^{k+d+1} d \lambda .
$$




\section{Proof of Lemma 3}

The sufficient part $((i i) \Rightarrow(i))$ again follows by the Chetaev theorem: if there exist the defined function $V$ with such a $B_{\delta}$, then the system (1) is unstable [16].

To prove that $(i) \Rightarrow(i i)$ note that if the set $B_{\delta} \cup\{0\}$ is backward invariant, it means that any not invariant trajectory of the system (1) leaves the set in a finite time and there is no trajectory entering $B_{\delta}$. Considering system (1) in the backward time, i.e. $\dot{\mathbf{x}}=-\mathbf{f}(\mathbf{x})$, we make the set $B_{\delta} \cup\{0\}$ forward invariant. By assumptions the only invariant solution is at the origin, thus all trajectories into the forward invariant set $B_{\delta} \cup\{0\}$ converge asymptotically to the equilibrium $\mathbf{x}=0$ in the backward time. In [38] (Theorem 1.5.40) it is shown that under these conditions the system is asymptotically stable at the origin into the set $B_{\delta} \cup\{0\}$. Let us show that in this case there exists a locally Lipschitz continuous function $V: B_{\delta} \cup\{0\} \rightarrow R_{+}$(continuous at the origin) such that

$$
\alpha_{1}(|\mathbf{x}|) \leq V(\mathbf{x}) \leq \alpha_{2}(|\mathbf{x}|), \alpha_{1}, \alpha_{2} \in \mathcal{K}_{\infty}, L_{-\mathbf{f}(\mathbf{x})} V(\mathbf{x}) \leq-\alpha_{3}(V(\mathbf{x})), \alpha_{3} \in \mathcal{K}
$$

for a.e. $\mathbf{x} \in B_{\delta} \cup\{0\}$. For this purpose for any $\mathbf{x}_{0} \in B_{\delta} \cup\{0\}$ define

$$
v\left(\mathbf{x}_{0}\right)=\sup _{t \geq 0}\left|\mathbf{x}\left(t, \mathbf{x}_{0}\right)\right|
$$

by construction $\left|\mathbf{x}_{0}\right| \leq v\left(\mathbf{x}_{0}\right) \leq \phi\left(\left|\mathbf{x}_{0}\right|\right) \leq \delta, \phi(s)=(1+s) \sup _{|\mathbf{x}| \leq s, \mathbf{x} \in B_{\delta}} v(\mathbf{x})$ and $v(0)=0$. From attractivity for any $\mathbf{x}_{0} \in B_{\delta}$ there exists $T_{\mathbf{x}_{0}} \in R_{+}$such that $v\left(\mathbf{x}_{0}\right)=\sup _{0 \leq t \leq T_{\mathbf{x}_{0}}}\left|\mathbf{x}\left(t, \mathbf{x}_{0}\right)\right|$. To analyze continuity property of the function $v$ consider

$$
\begin{aligned}
\left|v\left(\mathbf{x}_{1}\right)-v\left(\mathbf{x}_{2}\right)\right| & =\left|\sup _{t \geq 0}\right| \mathbf{x}\left(t, \mathbf{x}_{1}\right)\left|-\sup _{t \geq 0}\right| \mathbf{x}\left(t, \mathbf{x}_{2}\right) \| \\
& =\left|\sup _{0 \leq t \leq T_{\mathbf{x}_{1}}}\right| \mathbf{x}\left(t, \mathbf{x}_{1}\right)\left|-\sup _{0 \leq t \leq T_{\mathbf{x} 2}}\right| \mathbf{x}\left(t, \mathbf{x}_{2}\right) \| \\
& \leq \sup _{0 \leq t \leq T}\left\|\mathbf{x}\left(t, \mathbf{x}_{1}\right)|-| \mathbf{x}\left(t, \mathbf{x}_{2}\right)\right\|,
\end{aligned}
$$

where $T=\max \left\{T_{\mathbf{x}_{1}}, T_{\mathbf{x}_{2}}\right\}, \mathbf{x}_{1}, \mathbf{x}_{2} \in B_{\delta}$. Due to Lipschitz continuity of the system (1) solutions on any compact set of initial conditions $\mathcal{D} \subset B_{\delta}$ and time $0 \leq T<+\infty$ there exists $L \in R_{+}$such that

$$
\left|\mathbf{x}\left(t, \mathbf{x}_{1}\right)-\mathbf{x}\left(t, \mathbf{x}_{2}\right)\right| \leq L\left|\mathbf{x}_{1}-\mathbf{x}_{2}\right|
$$

for all $0 \leq t \leq T$ and any $\mathbf{x}_{1}, \mathbf{x}_{2} \in \mathcal{D}$. For all $0<\delta^{\prime} \leq \delta$ for any compact $\mathcal{D} \subset B_{\delta}$ there exists $T_{\delta^{\prime}}=\sup _{\mathbf{x}_{0} \in \mathcal{D} \backslash B_{\delta^{\prime}}} T_{\mathbf{x}_{0}}$ with the property $T_{\delta^{\prime}}<+\infty$, then

$$
\left|v\left(\mathbf{x}_{1}\right)-v\left(\mathbf{x}_{2}\right)\right| \leq \sup _{0 \leq t \leq T_{\delta^{\prime}}}|| \mathbf{x}\left(t, \mathbf{x}_{1}\right)|-| \mathbf{x}\left(t, \mathbf{x}_{2}\right)|| \leq L\left|\mathbf{x}_{1}-\mathbf{x}_{2}\right|
$$

for all $\mathbf{x}_{1}, \mathbf{x}_{2} \in \mathcal{D} \backslash B_{\delta^{\prime}}$ and the function $v$ is locally Lipschitz continuous on the set $\mathcal{D} \backslash B_{\delta^{\prime}}$ for any fixed $0<\delta^{\prime} \leq \delta$. The function $v$ is not increasing on any trajectory of the system (1), indeed for any $\mathbf{x}_{0} \in B_{\delta} \cup\{0\}$ :

$$
v\left(\mathbf{x}\left(t, \mathbf{x}_{0}\right)\right)=\sup _{\tau \geq 0}\left|\mathbf{x}\left(\tau, \mathbf{x}\left(t, \mathbf{x}_{0}\right)\right)\right|=\sup _{\tau \geq t}\left|\mathbf{x}\left(\tau, \mathbf{x}_{0}\right)\right| \leq \sup _{\tau \geq 0}\left|\mathbf{x}\left(\tau, \mathbf{x}_{0}\right)\right|=v\left(\mathbf{x}_{0}\right)
$$


To construct a strictly decreasing function, define a new function for all $\mathbf{x}_{0} \in B_{\delta} \cup\{0\}$ :

$$
V\left(\mathbf{x}_{0}\right)=\sup _{t \geq 0}\left\{v\left(\mathbf{x}\left(t, \mathbf{x}_{0}\right)\right) k(t)\right\}
$$

where $k: R_{+} \rightarrow R_{+}$is a continuously differentiable function with the properties $0<\kappa_{1} \leq k(t) \leq \kappa_{2}<+\infty$ and $\dot{k}(t) \geq \kappa_{3}(t)>0$ for all $t \geq 0$ [27]. An example of such function is as follows:

$$
k(t)=\left(\kappa_{1}+\kappa_{2} t^{1+\omega}\right)\left(1+t^{1+\omega}\right)^{-1}, \dot{k}(t)=t^{\omega}\left(\kappa_{2}-\kappa_{1}\right)(1+\omega)\left(1+t^{1+\omega}\right)^{-2}, \omega \geq 0 .
$$

The function $V$ has bounds $\kappa_{1}\left|\mathbf{x}_{0}\right| \leq V\left(\mathbf{x}_{0}\right) \leq \kappa_{2} \phi\left(\left|\mathbf{x}_{0}\right|\right)$ and $V(0)=0$. Again, for any $\mathbf{x}_{0} \in B_{\delta} \cup\{0\}$ there exists $T_{\mathbf{x}_{0}} \in R+$ such that $V\left(\mathbf{x}_{0}\right)=\sup _{0 \leq t \leq T_{\mathbf{x}_{0}}}\left\{v\left(\mathbf{x}\left(t, \mathbf{x}_{0}\right)\right) k(t)\right\}$. This claim follows from non strict decreasing of the function $v$. Next, for all $\mathbf{x}_{1}, \mathbf{x}_{2} \in B_{\delta}$

$$
\begin{aligned}
\left|V\left(\mathbf{x}_{1}\right)-V\left(\mathbf{x}_{2}\right)\right| & =\left|\sup _{t \geq 0}\left\{v\left(\mathbf{x}\left(t, \mathbf{x}_{1}\right)\right) k(t)\right\}-\sup _{t \geq 0}\left\{v\left(\mathbf{x}\left(t, \mathbf{x}_{2}\right)\right) k(t)\right\}\right| \\
& =\left|\sup _{0 \leq t \leq T}\left\{v\left(\mathbf{x}\left(t, \mathbf{x}_{1}\right)\right) k(t)\right\}-\sup _{0 \leq t \leq T}\left\{v\left(\mathbf{x}\left(t, \mathbf{x}_{2}\right)\right) k(t)\right\}\right| \\
& \leq \sup _{0 \leq t \leq T}\left|k(t)\left[v\left(\mathbf{x}\left(t, \mathbf{x}_{1}\right)\right)-v\left(\mathbf{x}\left(t, \mathbf{x}_{2}\right)\right)\right]\right| \\
& \leq \kappa_{2} \sup _{0 \leq t \leq T}\left|v\left(\mathbf{x}\left(t, \mathbf{x}_{1}\right)\right)-v\left(\mathbf{x}\left(t, \mathbf{x}_{2}\right)\right)\right|
\end{aligned}
$$

where $T=\max \left\{T_{\mathbf{x}_{1}}, T_{\mathbf{x}_{2}}\right\}$. For all $0<\delta^{\prime} \leq \delta$ for any compact $\mathcal{D} \subset B_{\delta}$ there exists $T_{\delta^{\prime}}=\sup _{\mathbf{x}_{0} \in \mathcal{D} \backslash B_{\delta^{\prime}}} T_{\mathbf{x}_{0}}$ with the property $T_{\delta^{\prime}}<+\infty$ and

$$
\begin{aligned}
\left|V\left(\mathbf{x}_{1}\right)-V\left(\mathbf{x}_{2}\right)\right| & \leq \kappa_{2} \sup _{0 \leq t \leq T_{\delta^{\prime}}}\left|v\left(\mathbf{x}\left(t, \mathbf{x}_{1}\right)\right)-v\left(\mathbf{x}\left(t, \mathbf{x}_{2}\right)\right)\right| \\
& \leq \kappa_{2} L\left|\mathbf{x}\left(t, \mathbf{x}_{1}\right)-\mathbf{x}\left(t, \mathbf{x}_{2}\right)\right| \leq \kappa_{2} L^{2}\left|\mathbf{x}_{1}-\mathbf{x}_{2}\right|
\end{aligned}
$$

for all $\mathbf{x}_{1}, \mathbf{x}_{2} \in \mathcal{D} \backslash B_{\delta^{\prime}}$. Then the function $V$ is locally Lipschitz continuous on the set $\mathcal{D} \backslash B_{\delta^{\prime}}$ for any $0<\delta^{\prime} \leq \delta$ and it is strictly decreasing for any $\mathbf{x}_{0} \in B_{\delta}$ :

$$
\begin{aligned}
V\left(\mathbf{x}\left(t, \mathbf{x}_{0}\right)\right) & =\sup _{\tau \geq 0}\left\{v\left(\mathbf{x}\left[\tau, \mathbf{x}\left(t, \mathbf{x}_{0}\right)\right]\right) k(\tau)\right\}=\sup _{\tau \geq t}\left\{v\left(\mathbf{x}\left[\tau, \mathbf{x}_{0}\right]\right) k(\tau-t)\right\} \\
& <\sup _{\tau \geq 0}\left\{v\left(\mathbf{x}\left[\tau, \mathbf{x}_{0}\right]\right) k(\tau)\right\}=V\left(\mathbf{x}_{0}\right)
\end{aligned}
$$

$V(t)$ is zero on the trajectories at the origin. Denote $L_{-\mathbf{f}\left(\mathbf{x}_{0}\right)} V\left(\mathbf{x}_{0}\right)=\lim _{h \rightarrow 0} h^{-1}\left[V\left(\mathbf{x}\left(h, \mathbf{x}_{0}\right)\right)-V\left(\mathbf{x}_{0}\right)\right]$, then

$$
L_{-\mathbf{f}\left(\mathbf{x}_{0}\right)} V\left(\mathbf{x}_{0}\right)<0
$$

for a.e. $\mathbf{x}_{0} \in B_{\delta}$. Denote $T_{\delta}=\sup _{\mathbf{x}_{0} \in B_{\delta}} T_{\mathbf{x}_{0}}$ and by the definition

$$
\begin{aligned}
V\left(\mathbf{x}\left(h, \mathbf{x}_{0}\right)\right) & =\sup _{t \geq 0}\left\{v\left(\mathbf{x}\left[t, \mathbf{x}\left(h, \mathbf{x}_{0}\right)\right]\right) k(t)\right\} \\
& =\sup _{h \leq t \leq T_{\delta}}\left\{v\left(\mathbf{x}\left[t, \mathbf{x}_{0}\right]\right) k(t-h)\right\}=\sup _{0 \leq t \leq T_{\delta}}\left\{v\left(\mathbf{x}\left[t, \mathbf{x}_{0}\right]\right) K(t, h)\right\}
\end{aligned}
$$


for a.e. $\mathbf{x}_{0} \in B_{\delta}$ and $K(t, h)=i f[t<h, 0, k(t-h)]$, further

$$
\begin{aligned}
\sup _{0 \leq t \leq T_{\delta}}\left\{v\left(\mathbf{x}\left[t, \mathbf{x}_{0}\right]\right) K(t, h)\right\} & \leq V\left(\mathbf{x}_{0}\right) \sup _{0 \leq t \leq T_{\delta}}\left\{k(t)^{-1} K(t, h)\right\} \\
& =V\left(\mathbf{x}_{0}\right) \max \left\{0, \sup _{h \leq t \leq T_{\delta}}\left\{k(t)^{-1} k(t-h)\right\}\right\} \\
& =V\left(\mathbf{x}_{0}\right) \sup _{h \leq t \leq T_{\delta}}\left\{k(t)^{-1} k(t-h)\right\}
\end{aligned}
$$

and

$$
\begin{aligned}
\lim _{h \rightarrow 0} h^{-1}\left[V\left(\mathbf{x}\left(h, \mathbf{x}_{0}\right)\right)-V\left(\mathbf{x}_{0}\right)\right] & =\lim _{h \rightarrow 0} h^{-1}\left[V\left(\mathbf{x}_{0}\right) \sup _{h \leq t \leq T_{\delta}}\left\{k(t)^{-1} k(t-h)\right\}-V\left(\mathbf{x}_{0}\right)\right] \\
& =V\left(\mathbf{x}_{0}\right) \lim _{h \rightarrow 0} h^{-1}\left[\sup _{h \leq t \leq T_{\delta}}\left\{k(t)^{-1} k(t-h)\right\}-1\right] \\
& =V\left(\mathbf{x}_{0}\right) \lim _{h \rightarrow 0} h^{-1} \sup _{h \leq t \leq T_{\delta}} k(t)^{-1}\{k(t-h)-k(t)\} \\
& \leq V\left(\mathbf{x}_{0}\right) \sup _{0 \leq t \leq T_{\delta}} k(t)^{-1} \lim _{h \rightarrow 0} h^{-1}\{k(t-h)-k(t)\} \\
& =V\left(\mathbf{x}_{0}\right) \sup _{t \geq 0}\left\{-k(t)^{-1} \dot{k}(t)\right\} \leq-\kappa_{2}^{-1} \kappa_{3}\left(T_{\delta}\right) V\left(\mathbf{x}_{0}\right) .
\end{aligned}
$$

We substantiate the inequality

$$
L_{-\mathbf{f}(\mathbf{x})} V(\mathbf{x}) \leq-\kappa_{2}^{-1} \kappa_{3}\left(T_{\delta}\right) V(\mathbf{x})
$$

for a.e. $\mathbf{x} \in B_{\delta} \cup\{0\}$ (the inequality is additionally valid at the origin), $\alpha_{3}(s)=\kappa_{2}^{-1} \kappa_{3}\left(T_{\delta}\right) s$ and $\alpha_{1}(s)=\kappa_{1} s, \alpha_{2}(s)=$ $\kappa_{2} \phi(s)$. Existence of a continuously differentiable function $V$ can be substantiated applying standard smoothing arguments [27]. Returning to the initial forward time we obtain existence of the required continuously differentiable Chetaev function for the system (1) in $B_{\delta} \cup\{0\}$. To prove existence of a homogeneous Chetaev function for $\mathbf{r}$-homogeneous function $\mathbf{f}$ one can apply the same arguments as in the proof of Lemma 2.

\section{Proof of Proposition 1}

$1 \Rightarrow 2$. Application of the dilation transformation for the $\left(\mathbf{r}, \lambda_{0}, \mathbf{f}_{0}\right)$-homogeneous system (1) gives:

$$
\begin{aligned}
D V_{0}(\mathbf{x}) \mathbf{f}(\mathbf{x}) & =D V_{0}\left(\boldsymbol{\Lambda}_{\mathbf{r}} \mathbf{y}\right) \mathbf{f}\left(\boldsymbol{\Lambda}_{\mathbf{r}} \mathbf{y}\right) \\
& =D V_{0}\left(\boldsymbol{\Lambda}_{\mathbf{r}} \mathbf{y}\right) \lambda^{d} \boldsymbol{\Lambda}_{\mathbf{r}} \mathbf{f}_{0}(\mathbf{y})+D V_{0}\left(\boldsymbol{\Lambda}_{\mathbf{r}} \mathbf{y}\right)\left[\mathbf{f}\left(\boldsymbol{\Lambda}_{\mathbf{r}} \mathbf{y}\right)-\lambda^{d} \boldsymbol{\Lambda}_{\mathbf{r}} \mathbf{f}_{0}(\mathbf{y})\right] \\
& =\lambda^{d+k} D V_{0}(\mathbf{y}) \mathbf{f}_{0}(\mathbf{y})+\lambda^{d+k} D V_{0}(\mathbf{y})\left[\lambda^{-d} \boldsymbol{\Lambda}_{\mathbf{r}}^{-1} \mathbf{f}\left(\boldsymbol{\Lambda}_{\mathbf{r}} \mathbf{y}\right)-\mathbf{f}_{0}(\mathbf{y})\right]
\end{aligned}
$$

where $k$ and $d$ are the homogeneity degree of the Lyapunov function $V_{0}$ and the function $\mathbf{f}_{0}$ respectively, $\mathbf{x} \in R^{n}, \mathbf{y} \in S_{\mathbf{r}}$. Due to continuity of the functions $\mathbf{f}, \mathbf{f}_{0}$ and the local homogeneity property definition, for any $\varepsilon>0$ there exist $\underline{\lambda}_{\varepsilon}(\mathbf{y}) \leq \lambda_{0}(\mathbf{y}) \leq \bar{\lambda}_{\varepsilon}(\mathbf{y})$ such that

$$
\sup _{\lambda \in\left(\lambda_{\varepsilon}(\mathbf{y}), \bar{\lambda}_{\varepsilon}(\mathbf{y})\right), \mathbf{y} \in S_{\mathbf{r}}}\left|D V_{0}(\mathbf{y})\left[\lambda^{-d} \boldsymbol{\Lambda}_{\mathbf{r}}^{-1} \mathbf{f}\left(\boldsymbol{\Lambda}_{\mathbf{r}} \mathbf{y}\right)-\mathbf{f}_{0}(\mathbf{y})\right]\right| \leq \varepsilon .
$$

Therefore, for the properly chosen $\underline{\lambda}_{\varepsilon}, \bar{\lambda}_{\varepsilon}$ the sign of $D V_{0}(\mathbf{y}) \mathbf{f}_{0}(\mathbf{y})$ can override the sign of $D V_{0}(\mathbf{x}) \mathbf{f}(\mathbf{x})$. Depending on this sign the set $\mathcal{X}$ can be (locally) asymptotically stable or unstable. 
$2 \Rightarrow 1$. According to definition of $\left(\mathbf{r}, \lambda_{0}, \mathbf{f}_{0}\right)$-homogeneity, the functions $\mathbf{f}_{0}$ and $\mathbf{f}$ coincide on the set $\mathcal{S}$ and $\mathbf{f}_{0}$ is the unique local approximation of $\mathbf{f}$ for the given $d, d_{0}, \mathbf{r}$ and $\lambda_{0}$. Since the function $\mathbf{f}_{0}$ is homogeneous it may be globally stable or unstable at the origin in this case, actually applying the same dilation transformation but in the inverse direction we can show that for $\mathbf{x} \in \mathcal{S}$ the sign of $D V_{0}(\mathbf{x}) \mathbf{f}(\mathbf{x})$ overrides the sign of $D V_{0}(\mathbf{x}) \mathbf{f}_{0}(\mathbf{x})$, since both $V_{0}$ and $\mathbf{f}_{0}$ are homogeneous the result follows.

\section{Proof of Proposition 2}

As in the proof of Proposition 1, since $\mathcal{S}=\left\{\boldsymbol{\Lambda}_{\mathbf{r}, 0}(\mathbf{y}) \mathbf{y}, \mathbf{y} \in S_{\mathbf{r}}\right\}=\left\{\mathbf{x} \in R^{n}: V_{0}(\mathbf{x})=b\right\}$, then it is possible to select $\underline{\lambda}_{\varepsilon}$, $\bar{\lambda}_{\varepsilon}$ (the relations (10) are satisfied for $\underline{\lambda}_{\varepsilon}(\mathbf{y}) \leq \lambda_{0}(\mathbf{y}) \leq \bar{\lambda}_{\varepsilon}(\mathbf{y})$ for all $\mathbf{y} \in S_{\mathbf{r}}$ ) in such a way that the sign of $D V_{0}(\mathbf{x}) \mathbf{f}(\mathbf{x})$ for $\mathbf{x} \in X$ is predefined by $a_{z}, z \in\{s, u\}$.

Consider the case (i), then $a_{s}>0$ and for all $\mathbf{x} \in X$

$$
D V_{0}(\mathbf{x}) \mathbf{f}(\mathbf{x}) \leq-\lambda^{d+k}\left(a_{s}-\varepsilon\right)=-|\mathbf{x}|_{\mathbf{r}}^{d+k}\left(a_{s}-\varepsilon\right)
$$

If $\lambda_{0}=0$, then clearly $b=0$ and the system is locally asymptotically stable at the origin with the domain of asymptotic stability containing the set $X_{0}$ for some $0<\epsilon<+\infty$ by the standard arguments [26]. If $\lambda_{0}=+\infty$, then the function $V_{0}$ has strictly negative time derivative for the system (1) into the set $R^{n} \backslash X_{0}$ for some $0<\epsilon<+\infty$. Thus the set $X_{0}$ is forward invariant for (1) and according to [27] these facts imply the global asymptotic stability of the system (1) with respect to the set $X_{0}$. Finally, let $0<\lambda_{0}(\mathbf{x})<+\infty$, then the function $V_{0}$ is strictly decreasing into the set $X$ and all trajectories $\mathbf{x}\left(t, \mathbf{x}_{0}\right)$ with initial conditions $\mathbf{x}_{0} \in X$ reach for the set $X_{1}$ in a finite time, which implies the desired conclusion.

Consider the case (ii), then $a_{u}>0$ and

$$
D V_{0}(\mathbf{x}) \mathbf{f}(\mathbf{x}) \geq \lambda^{d+k}\left(a_{u}-\varepsilon\right)=|\mathbf{x}|_{\mathbf{r}}^{d+k}\left(a_{u}-\varepsilon\right)
$$

for all $\mathrm{x} \in X$. If $\lambda_{0}=0$, then the system is unstable into the set $X_{0}$ by the standard arguments [26]. Therefore, the set $R^{n} \backslash X_{0}$ is forward invariant and has the region of attraction $X_{0} \backslash\{0\}$. If $\lambda_{0}=+\infty$, then the function $V_{0}$ has a strictly positive time derivative for the system (1) into the set $R^{n} \backslash X_{0}$ for some $0<\epsilon<+\infty$. Thus the set $R^{n} \backslash X_{0}$ is forward invariant for (1). Finally, let $0<\lambda_{0}(\mathbf{x})<+\infty$, then the function $V_{0}$ is strictly increasing into the set $X$ and all trajectories $\mathbf{x}\left(t, \mathbf{x}_{0}\right)$ with initial conditions $\mathbf{x}_{0} \in X$ reach for the set $X_{2}$ in a finite time, which implies forward invariance of this set and finite time stability with the region of attraction $X$.

\section{Proof of Corollary 1}

Under the corollary conditions the time derivative of the function $V_{0}$ for the system (1) has the same form:

$$
D V_{0}(\mathbf{x}) \mathbf{f}(\mathbf{x})=\lambda^{d+k} D V_{0}(\mathbf{y}) \mathbf{f}_{0}(\mathbf{y})+\lambda^{d+k} D V_{0}(\mathbf{y})\left[\lambda^{-d} \boldsymbol{\Lambda}_{\mathbf{r}}^{-1} \mathbf{f}\left(\boldsymbol{\Lambda}_{\mathbf{r}} \mathbf{y}\right)-\mathbf{f}_{0}(\mathbf{y})\right]
$$

And for the cases (i) and (ii) the inequalities

$$
D V_{0}(\mathbf{x}) \mathbf{f}(\mathbf{x}) \leq-|\mathbf{x}|_{\mathbf{r}}^{d+k}(a-\varepsilon), D V_{0}(\mathbf{x}) \mathbf{f}(\mathbf{x}) \geq|\mathbf{x}|_{\mathbf{r}}^{d+k}(a-\varepsilon)
$$


Further, the conclusion follows by the same arguments as in Proposition 2.

\section{Proof of Proposition 3}

As in Proposition 2, transformation to the sphere $S_{\mathbf{r}}$ for the $\left(\mathbf{r}, \lambda_{0}, \mathbf{f}_{0}\right)$-homogeneous system (1) gives:

$$
D V_{0}(\mathbf{x}) \mathbf{f}(\mathbf{x})=\lambda^{d+k} D V_{0}(\mathbf{y}) \mathbf{f}_{0}(\mathbf{y})+\lambda^{d+k} D V_{0}(\mathbf{y})\left[\lambda^{-d} \boldsymbol{\Lambda}_{\mathbf{r}}^{-1} \mathbf{f}\left(\boldsymbol{\Lambda}_{\mathbf{r}} \mathbf{y}\right)-\mathbf{f}_{0}(\mathbf{y})\right]
$$

where $d$ and $k$ are the homogeneity degree of the Lyapunov function $V_{0}$ and the function $\mathbf{f}$ respectively. Due to continuity of the functions $\mathbf{f}, \mathbf{f}_{0}$ and the local homogeneity property definition for any $\varepsilon>0$ there exists $0<\bar{\lambda}_{\varepsilon}$ such that

$$
\sup _{\mathbf{y} \in S_{\mathbf{r}}}\left|D V_{0}(\mathbf{y})\left[\lambda^{-d} \boldsymbol{\Lambda}_{\mathbf{r}}^{-1} \mathbf{f}\left(\boldsymbol{\Lambda}_{\mathbf{r}} \mathbf{y}\right)-\mathbf{f}_{0}(\mathbf{y})\right]\right| \leq \varepsilon
$$

for all $\lambda \in\left(0, \bar{\lambda}_{\varepsilon}\right)$. Therefore, for the properly chosen $\bar{\lambda}_{\varepsilon}$ the sign of $D V_{0}(\mathbf{y}) \mathbf{f}_{0}(\mathbf{y})$ can override the sign of $D V_{0}(\mathbf{x}) \mathbf{f}(\mathbf{x})$ into the set $S_{\mathbf{r}} \cap K$ provided that $\varepsilon<a$ :

$$
D V_{0}(\mathbf{x}) \mathbf{f}(\mathbf{x}) \geq \lambda^{d+k}(a-\varepsilon)=|\mathbf{x}|_{\mathbf{r}}^{d+k}(a-\varepsilon)
$$

for all $\mathbf{x} \in X_{\mathbf{r}}=\left\{\mathbf{x} \in K: 0<|\mathbf{x}|_{\mathbf{r}}<\bar{\lambda}_{\varepsilon}\right\}$. Therefore, the system is unstable at the origin [16].

\section{Proof of Theorem 6}

For the part $(i)$ the existence of such an index $j^{*}$ means that $\mathbf{f}_{j^{*}}$ is unstable and the vector field $\mathbf{f}_{j^{*}+1}$ is asymptotically stable. Thus, from part $(i i)-3$ and part $(i)-3$ of Proposition 2 , there exist $\underline{\lambda}_{k} \leq \lambda_{k} \leq \bar{\lambda}_{k}$, for $k=j^{*}, j^{*}+1$ such that the Lyapunov functions $V_{k}, k=j^{*}, j^{*}+1$ have sign definite time derivatives for the system (1) into the sets

$$
X_{\mathbf{r}_{k}}=\left\{\mathbf{x} \in R^{n}: \underline{\lambda}_{k}<|\mathbf{x}|_{\mathbf{r}_{k}}<\bar{\lambda}_{k}\right\}, k=j^{*}, j^{*}+1
$$

respectively. Since the sets $X_{k}, k=j *, j *+1$ are connected and nonempty, then all trajectories of the system (1) with initial conditions into the set $\Omega_{j *}$ stay there for all further instants of time. By the same arguments all trajectories of the system (1) with initial conditions into the set $\Omega_{j^{*}+1}$ stay there. Therefore, the set $\Omega=\Omega_{j *} \cap \Omega_{j *+1}$ being nonempty is forward invariant and compact. By the theorem conditions this set does not contain the system (1) equilibria $(\Omega \cap \Xi=\emptyset)$.

Consider any trajectory $\mathbf{x}\left(t, \mathbf{x}_{0}\right)$ of the system (1) with initial conditions $\mathbf{x}_{0} \in \Omega$, by consideration above $\mathbf{x}\left(t, \mathbf{x}_{0}\right) \in \Omega$ for all $t \geq 0$ and the trajectories $\mathbf{x}\left(t, \mathbf{x}_{0}\right)$ are bounded due to compactness of $\Omega$. Then for each $\mathbf{x}_{0} \in \Omega$ there should exist an index $1 \leq i^{*} \leq n$ such that the solution $\mathbf{x}\left(t, \mathbf{x}_{0}\right)$ is $\left[\pi^{-}, \pi^{+}\right]$-oscillation with respect to the output $\psi=x_{i *}$ for some $-\infty<\pi^{-}<\pi^{+}<+\infty$. Indeed, the definition

$$
\pi^{-}=\lim \inf _{t \rightarrow+\infty} \psi(t), \pi^{+}=\lim \sup _{t \rightarrow+\infty} \psi(t)
$$

and the boundedness of the trajectory $\mathbf{x}\left(t, \mathbf{x}_{0}\right)$ imply that the constants $\pi^{-}, \pi^{+}$are finite. If for all $1 \leq i^{*} \leq n$ for the corresponding constants the quantity $\pi^{-}=\pi^{+}$holds, then it means that there is $\mathbf{x}_{\infty} \in \Omega$ such that $\lim _{t \rightarrow+\infty} \mathbf{x}\left(t, \mathbf{x}_{0}\right)=\mathbf{x}_{\infty}$, which is a contradiction since $\mathbf{x}_{\infty}$ is an equilibrium and all equilibria are excluded from $\Omega$. 
For the part $(i i), \mathbf{f}_{j^{*}}$ is stable and the vector field $\mathbf{f}_{j^{*+1}}$ is unstable. Again, according to Proposition 2 there exist $\underline{\lambda}_{k} \leq \lambda_{k} \leq$ $\bar{\lambda}_{k}$, for $k=j^{*}, j^{*}+1$ such that the Lyapunov functions $V_{k}, k=j^{*}, j^{*}+1$ have sign definite time derivatives for the system (1) into the sets $X_{\mathbf{r}_{k}}, k=j^{*}, j^{*}+1$. Since the sets $X_{k}, k=j *, j *+1$ are connected and nonempty, then all trajectories of the system (1) with initial conditions into the sets $R^{n} \backslash \Omega_{j^{*}}$ and $R^{n} \backslash \Omega_{j^{*}+1}$ stay there for all positive times. Therefore, the complement set $\Omega$ being nonempty is backward invariant and compact. By the theorem conditions this set does not contain the system (1) equilibria. Since the system (1) is time-invariant, reversing the time we transform the set $\Omega$ to forward invariant and compact set for the system $\dot{\mathbf{x}}=-\mathbf{f}(\mathbf{x})$. Applying to this system the same arguments as in the part $(i)$ we can prove existence of oscillating trajectories into the set $\Omega$, which implies the same conclusion for the original system (1). 